TRANSACTIONS OF THE

AMERICAN MATHEMATICAL SOCIETY

Volume 361, Number 5, May 2009, Pages 2689-2718

S 0002-9947(08)04631-X

Article electronically published on November 20, 2008

\title{
ROUGH PATH ANALYSIS VIA FRACTIONAL CALCULUS
}

\author{
YAOZHONG HU AND DAVID NUALART
}

\begin{abstract}
Using fractional calculus we define integrals of the form $\int_{a}^{b} f\left(x_{t}\right) d y_{t}$, where $x$ and $y$ are vector-valued Hölder continuous functions of order $\beta \in$ $\left(\frac{1}{3}, \frac{1}{2}\right)$ and $f$ is a continuously differentiable function such that $f^{\prime}$ is $\lambda$-Hölder continuous for some $\lambda>\frac{1}{\beta}-2$. Under some further smooth conditions on $f$ the integral is a continuous functional of $x, y$, and the tensor product $x \otimes y$ with respect to the Hölder norms. We derive some estimates for these integrals and we solve differential equations driven by the function $y$. We discuss some applications to stochastic integrals and stochastic differential equations.
\end{abstract}

\section{INTRODUCTION}

The theory of rough path analysis has been developed from the seminal paper by Lyons 10 . The purpose of this theory is to analyze $d$-dimensional dynamical systems $d x_{t}=f\left(x_{t}\right) d y_{t}$, where the control function $y$ is not differentiable. The natural assumption is that the control function $y$ has bounded $p$-variation for some $p>1$. If $1<p<2$, the dynamical system can be formulated using RiemannStieltjes integrals and the theory developed by Young in [17. In this case, $x$ is a continuous functional of $y$ in the $p$-variation norm (see Lyons [9]).

In the case $p \geq 2$, the main idea of rough path analysis is to lift $y$ to an element of the extended tensor algebra $T=\bigoplus_{n \geq 0}\left(\mathbb{R}^{d}\right)^{\otimes n}$ called the signature of $y$, defined by

$$
S(y)_{s, t}=\left(1, y_{s, t}^{1}, y_{s, t}^{2}, \ldots\right),
$$

where $y_{s, t}^{n}$ denotes the $n$th iterated integral of $y$ on the interval $[s, t] \subset[0, T]$. This signature satisfies the following multiplicativity property with respect to the tensor product on $T: S(y)_{s, t}=S(y)_{s, u} \otimes S(y)_{u, t}$ for any $s \leq u \leq t$. Then, a multiplicative functional of degree $n$ is said to have finite $p$-variation if

$$
\sup _{0 \leq i \leq n} \sup _{\pi} \sum\left|y_{t_{k}, t_{k+1}}^{i}\right|^{\frac{p}{i}}<\infty
$$

Received by the editors October 2, 2006 and, in revised form, September 6, 2007.

2000 Mathematics Subject Classification. Primary 60H10, 60H05; Secondary 26A42, 26A33, $46 \mathrm{E} 35$.

Key words and phrases. Rough path, fractional calculus, integral, integration by parts, differential equation, stability, stochastic differential equation, Wong-Zakai approximation, convergence rate.

The work of the first author was supported in part by the National Science Foundation under Grant No. DMS0204613 and DMS0504783.

The work of the second author was partially supported by the MCyT Grant BFM2000-0598 and the NSF Grant No. DMS-0604207.

(C)2008 American Mathematical Society Reverts to public domain 28 years from publication 
where $\pi: 0=t_{0}<t_{1}<\cdots<t_{n}=T$ runs over all partitions of $[0, T]$. It turns out that a multiplicative functional with finite $p$-variation is determined by its truncation at level $[p]$, and it is called a $p$-rough path. For instance, if $2 \leq p<3$, then a $p$-rough path is determined by $y^{1}$ and $y^{2}$. The dynamical system $d x_{t}=$ $f\left(x_{t}\right) d y_{t}$ can be extended to $p$-rough paths, and the main result of this theory is the continuity in the $p$-variation metric of the mapping $S(y) \mapsto S(x)$. There is an extensive literature on rough path analysis, which includes the reference book by Lyons and Qian [13, the review paper by Lejay 8], the Saint Flour lecture notes by Lyons [11] and the more algebraic approach presented by Gubinelli in [5]. Flow equations on spaces of rough paths are studied in [12.

Rough path analysis provides a path-wise approach to classical stochastic calculus, and this has been one of the motivations to build this theory. We refer, for instance, to [1], 3], 4] and [7] for recent applications of the rough path analysis to the stochastic calculus with respect to the Wiener process. A natural application of the rough path analysis is the stochastic calculus with respect to fractional Brownian motion with Hurst parameter $H \in(0,1)$. In [2] Coutin and Qian have applied rough path analysis to show the convergence of Wong-Zakai approximations for stochastic differential equations driven by a fractional Brownian motion with parameter $H \in\left(\frac{1}{4}, \frac{1}{2}\right)$. A large deviation principle has been obtained in 14 for these equations.

The purpose of this paper is to develop an alternative approach to the study of dynamical systems $d x_{t}=f\left(x_{t}\right) d y_{t}$, where the control function $y$ is Hölder continuous of order $\beta \in\left(\frac{1}{3}, \frac{1}{2}\right)$ (so, it has finite $p$-variation with $2<p<3$ ). This approach is based on the techniques of the classical fractional calculus and it has been inspired by the work of Nualart and Răşcanu [15] in the case $\beta>\frac{1}{2}$. In that case, $f(x)$ and $y$ are Hölder continuous of order larger than $\frac{1}{2}$, and the Riemann-Stieltjes integral $\int_{0}^{t} f\left(x_{s}\right) d y_{s}$ can be expressed as a Lebesgue integral using fractional derivatives (see Zähle [18] and Proposition 2.1 below). Further results have been obtained in [6] along the lines of the present paper.

Following the ideas in [18 and [15], we first provide in Section 3 an explicit formula for integrals of the form $\int_{a}^{b} f\left(x_{t}\right) d y_{t}$, where $x$ and $y$ are Hölder continuous of order $\beta \in\left(\frac{1}{3}, \frac{1}{2}\right)$. This formula, given in Theorem 3.1, is based on the fractional integration by parts formula, and it involves the functions $x, y$, and the quadratic multiplicative functional $x \otimes y$. It is important to remark that this explicit formula does not depend on any approximation scheme, and it allows us to derive estimates in the Hölder norm for the indefinite integral.

Section 4 is devoted to establishing the existence and uniqueness of a solution for the dynamical system $d x_{t}=f\left(x_{t}\right) d y_{t}$ driven by a Hölder continuous function $y$ of order $\beta \in\left(\frac{1}{3}, \frac{1}{2}\right)$. The main ingredient in the proof of these results is the explicit formula obtained in Section 3. This formula allows us to transform this equation into a system of integral equations for $x$ and $x \otimes y$ that can be solved by a standard application of a fixed point argument. We show how the solution depends continuously on the Hölder norm of $y$ and $y \otimes y$. We also prove some stability results for the differential equations.

The main differences with similar results obtained in the framework of the rough path analysis are the following:

i) We provide an explicit expression in terms of $x, y$ and $x \otimes y$ for the integrals of the form $\int_{0}^{t} f\left(x_{s}\right) d y_{s}$ using fractional calculus. This expression is not based 
on any approximation argument and it leads to precise Hölder estimates (see Propositions 3.4, 6.4 and Corollary 6.5).

ii) We transform the dynamical system $d x_{t}=f\left(x_{t}\right) d y_{t}$ into a closed system of equations involving only $x, x \otimes y$ and $x \otimes(y \otimes y)$, which can be solved by a classical fixed point argument.

These results can be applied to implement a path-wise approach to define stochastic integrals and to solve stochastic differential equations driven by a multidimensional Brownian motion. As an application of the deterministic results obtained for dynamical systems we derive a sharp rate of almost sure convergence of the Wong-Zakai approximation for multidimensional diffusion processes. We couldn't find these kinds of estimates elsewhere. Similar results hold in the case of a fractional Brownian motion with Hurst parameter $H \in\left(\frac{1}{3}, \frac{1}{2}\right)$; however they are more involved and will be treated in a forthcoming paper.

\section{Fractional integrals AND DeRivatives}

Let $a, b \in \mathbb{R}$ with $a<b$. Denote by $L^{p}(a, b), p \geq 1$, the space of Lebesgue measurable functions $f:[a, b] \rightarrow \mathbb{R}$ for which $\|f\|_{L^{p}(a, b)}<\infty$, where

$$
\|f\|_{L^{p}(a, b)}= \begin{cases}\left(\int_{a}^{b}|f(t)|^{p} d t\right)^{1 / p}, & \text { if } 1 \leq p<\infty \\ \operatorname{ess} \sup \{|f(t)|: t \in[a, b]\}, & \text { if } p=\infty .\end{cases}
$$

Let $f \in L^{1}(a, b)$ and $\alpha>0$. The left-sided and right-sided fractional RiemannLiouville integrals of $f$ of order $\alpha$ are defined for almost all $t \in(a, b)$ by

and

$$
I_{a+}^{\alpha} f(t)=\frac{1}{\Gamma(\alpha)} \int_{a}^{t}(t-s)^{\alpha-1} f(s) d s
$$

$$
I_{b-}^{\alpha} f(t)=\frac{(-1)^{-\alpha}}{\Gamma(\alpha)} \int_{t}^{b}(s-t)^{\alpha-1} f(s) d s
$$

respectively, where $(-1)^{-\alpha}=e^{-i \pi \alpha}$ and $\Gamma(\alpha)=\int_{0}^{\infty} r^{\alpha-1} e^{-r} d r$ is the Euler gamma function. Let $I_{a+}^{\alpha}\left(L^{p}\right)$ (resp. $\left.I_{b-}^{\alpha}\left(L^{p}\right)\right)$ be the image of $L^{p}(a, b)$ by the operator $I_{a+}^{\alpha}$ (resp. $I_{b-}^{\alpha}$ ). If $f \in I_{a+}^{\alpha}\left(L^{p}\right)$ (resp. $\left.f \in I_{b-}^{\alpha}\left(L^{p}\right)\right)$ and $0<\alpha<1$, then the Weyl derivatives are defined as

$$
D_{a+}^{\alpha} f(t)=\frac{1}{\Gamma(1-\alpha)}\left(\frac{f(t)}{(t-a)^{\alpha}}+\alpha \int_{a}^{t} \frac{f(t)-f(s)}{(t-s)^{\alpha+1}} d s\right)
$$

and

$$
D_{b-}^{\alpha} f(t)=\frac{(-1)^{\alpha}}{\Gamma(1-\alpha)}\left(\frac{f(t)}{(b-t)^{\alpha}}+\alpha \int_{t}^{b} \frac{f(t)-f(s)}{(s-t)^{\alpha+1}} d s\right),
$$

where $a \leq t \leq b$ (the convergence of the integrals at the singularity $s=t$ holds point-wise for almost all $t \in(a, b)$ if $p=1$ and moreover in the $L^{p}$-sense if $1<p<$ $\infty)$.

For any $\lambda \in(0,1)$, we denote by $C^{\lambda}(a, b)$ the space of $\lambda$-Hölder continuous functions on the interval $[a, b]$. Recall from [16] that we have:

- If $\alpha<\frac{1}{p}$ and $q=\frac{p}{1-\alpha p}$, then

$$
I_{a+}^{\alpha}\left(L^{p}\right)=I_{b-}^{\alpha}\left(L^{p}\right) \subset L^{q}(a, b) .
$$


- If $\alpha>\frac{1}{p}$, then

$$
I_{a+}^{\alpha}\left(L^{p}\right) \cup I_{b-}^{\alpha}\left(L^{p}\right) \subset C^{\alpha-\frac{1}{p}}(a, b) .
$$

We have the following composition formulas:

$$
D_{a+}^{\alpha} D_{a+}^{\beta}=D_{a+}^{\alpha+\beta}, \quad D_{b-}^{\alpha} D_{b-}^{\beta}=D_{b-}^{\alpha+\beta},
$$

and similar formulas hold for the fractional integral operator. The following inversion formulas hold:

$$
\begin{array}{ll}
I_{a+}^{\alpha}\left(D_{a+}^{\alpha} f\right)=f, & \forall f \in I_{a+}^{\alpha}\left(L^{p}\right), \\
I_{a-}^{\alpha}\left(D_{a-}^{\alpha} f\right)=f, & \forall f \in I_{a-}^{\alpha}\left(L^{p}\right),
\end{array}
$$

and

$$
D_{a+}^{\alpha}\left(I_{a+}^{\alpha} f\right)=f, \quad D_{a-}^{\alpha}\left(I_{a-}^{\alpha} f\right)=f, \quad \forall f \in L^{1}(a, b) .
$$

On the other hand, for any $f, g \in L^{1}(a, b)$ we have

$$
\int_{a}^{b} I_{a+}^{\alpha} f(t) g(t) d t=(-1)^{\alpha} \int_{a}^{b} f(t) I_{b-}^{\alpha} g(t) d t,
$$

and for $f \in I_{a+}^{\alpha}\left(L^{p}\right)$ and $g \in I_{a-}^{\alpha}\left(L^{p}\right)$ we have

$$
\int_{a}^{b} D_{a+}^{\alpha} f(t) g(t) d t=(-1)^{-\alpha} \int_{a}^{b} f(t) D_{b-}^{\alpha} g(t) d t .
$$

Suppose that $f \in C^{\lambda}(a, b)$ and $g \in C^{\mu}(a, b)$ with $\lambda+\mu>1$. Then, from the classical paper by Young [17, the Riemann-Stieltjes integral $\int_{a}^{b} f d g$ exists. The following proposition can be regarded as a fractional integration by parts formula, and provides an explicit expression for the integral $\int_{a}^{b} f d g$ in terms of fractional derivatives (see [18]).

Proposition 2.1. Suppose that $f \in C^{\lambda}(a, b)$ and $g \in C^{\mu}(a, b)$ with $\lambda+\mu>1$. Let $1-\mu<\alpha<\lambda$. Then the Riemann-Stieltjes integral $\int_{a}^{b} f d g$ exists and it can be expressed as

$$
\int_{a}^{b} f d g=(-1)^{\alpha} \int_{a}^{b} D_{a+}^{\alpha} f(t) D_{b-}^{1-\alpha} g_{b-}(t) d t
$$

where $g_{b-}(t)=g(t)-g(b)$.

\section{INTEGRATION OF ROUGH FUNCTIONS}

Fix $\frac{1}{3}<\beta<\frac{1}{2}$ and a time interval $[0, T]$. Following [10] we introduce the following definition.

Definition 3.1. We will say that $(x, y, x \otimes y)$ is an $(m, d)$-dimensional $\beta$-Hölder continuous multiplicative functional if $x:[0, T] \rightarrow \mathbb{R}^{m}$ and $y:[0, T] \rightarrow \mathbb{R}^{d}$ are $\beta$-Hölder continuous functions and $x \otimes y$ is a continuous function defined on $\Delta:=\{(s, t): 0 \leq s \leq t \leq T\}$ with values on $\mathbb{R}^{m} \otimes \mathbb{R}^{d}$ and $x \otimes y$ satisfies the following properties:

i) For all $s \leq u \leq t$ we have (multiplicative property)

$$
(x \otimes y)_{s, u}+(x \otimes y)_{u, t}+\left(x_{u}-x_{s}\right) \otimes\left(y_{t}-y_{u}\right)=(x \otimes y)_{s, t} .
$$


ii) For all $(s, t) \in \Delta$,

$$
\left|(x \otimes y)_{s, t}\right| \leq c|t-s|^{2 \beta} .
$$

If $x$ and $y$ are continuously differentiable functions, then

$$
(x \otimes y)_{s, t}^{i, j}=\int_{s<\xi<\eta<t} d x_{\xi}^{i} d y_{\eta}^{j},
$$

$i=1, \ldots, m, j=1, \ldots, d$, clearly defines a $\beta$-Hölder continuous multiplicative functional.

For any $(s, t) \in \Delta$ and any function $x:[0, T] \rightarrow \mathbb{R}^{m}$, the $\gamma$-Hölder norm of $x$ on the interval $[s, t]$ will be denoted by

$$
\|x\|_{s, t, \gamma}=\sup _{s \leq \theta<r \leq t} \frac{\left|x_{r}-x_{\theta}\right|}{|r-\theta|^{\gamma}} .
$$

Also if $g: \Delta \rightarrow \mathbb{R}^{N}$ we set

$$
\|g\|_{s, t, \gamma}=\sup _{s \leq \theta<r \leq t} \frac{|g(\theta, r)|}{|r-\theta|^{\gamma}}
$$

We also set $\|x\|_{\gamma}=\|x\|_{0, T, \gamma}$ and $\|g\|_{\gamma}=\|g\|_{0, T, \gamma}$. On the other hand, $\|\cdot\|_{s, t, \infty}$ will denote the supremum norm in the interval $[s, t]$.

We denote by $M_{m, d}^{\beta}(0, T)$ the space of all $(m, d)$-dimensional $\beta$-Hölder continuous multiplicative functionals. On $M_{m, d}^{\beta}(0, T)$ we introduce the following functional for any $(s, t) \in \Delta$ :

$$
\Phi_{s, t, \beta}(x, y)=\|x \otimes y\|_{s, t, 2 \beta}+\|x\|_{s, t, \beta}\|y\|_{s, t, \beta} .
$$

Moreover, if $(x, y, x \otimes y)$ and $(y, z, y \otimes z)$ belong to $M_{m, d}^{\beta}(0, T)$ we set

$\Phi_{s, t, \beta}(x, y, z)=\|y\|_{s, t, \beta}\|x\|_{s, t, \beta}\|z\|_{s, t, \beta}+\|z\|_{s, t, \beta}\|x \otimes y\|_{s, t, 2 \beta}+\|x\|_{s, t, \beta}\|y \otimes z\|_{s, t, 2 \beta}$.

We notice that if $(x, y, x \otimes y)$ and $(\widetilde{x}, y, \widetilde{x} \otimes y)$ belong to $M_{m, d}^{\beta}(0, T)$, then $(x-\widetilde{x}, y, x \otimes y-\widetilde{x} \otimes y)$ also belongs to $M_{m, d}^{\beta}(0, T)$, and we will write $(x-\widetilde{x}) \otimes y=$ $x \otimes y-\widetilde{x} \otimes y$. In the same way, $x \otimes(y-\widetilde{y})=x \otimes y-x \otimes \widetilde{y}$.

Let $f: \mathbb{R}^{m} \rightarrow \mathbb{R}^{d}$ be a continuously differentiable function such that $f^{\prime}$ is locally $\lambda$-Hölder continuous, where $\lambda>\frac{1}{\beta}-2$. Suppose that $(x, y, x \otimes y)$ is an $(m, d)$ dimensional $\beta$-Hölder continuous multiplicative functional. Our aim is to define the integral

$$
\int_{a}^{b} f\left(x_{r}\right) d y_{r}=\sum_{i=1}^{d} \int_{a}^{b} f_{i}\left(x_{r}\right) d y_{r}^{i}
$$

using fractional calculus.

Fix a real number $\alpha$ such that $1-\beta<\alpha<2 \beta$ and $\alpha<\frac{\lambda \beta+1}{2}$. This is possible because $3 \beta>1$ and $\frac{\lambda \beta+1}{2}>1-\beta$.

Notice first that the fractional integration by parts formula (2.9) cannot be used to define the integral (3.8) because the fractional derivative $D_{a+}^{\alpha} f(x)$ is not well defined under our hypotheses. For this reason we introduce the following 
compensated fractional derivative for $r \in[0, a]$ :

$$
\begin{aligned}
\widehat{D}_{a+}^{\alpha} f(x)(r) & =\frac{1}{\Gamma(1-\alpha)}\left(\frac{f\left(x_{r}\right)}{(r-a)^{\alpha}}\right. \\
& \left.+\alpha \int_{a}^{r} \frac{f\left(x_{r}\right)-f\left(x_{\theta}\right)-\sum_{i=1}^{m} \partial_{i} f\left(x_{\theta}\right)\left(x_{r}^{i}-x_{\theta}^{i}\right)}{(r-\theta)^{\alpha+1}} d \theta\right) .
\end{aligned}
$$

This derivative is well defined under our hypotheses, because there exists a constant $K$ such that for all $r, \theta \in[0, a], \theta<r$, we have

$$
\frac{\left|f\left(x_{r}\right)-f\left(x_{\theta}\right)-\sum_{i=1}^{m} \partial_{i} f\left(x_{\theta}\right)\left(x_{r}^{i}-x_{\theta}^{i}\right)\right|}{(r-\theta)^{\alpha+1}} \leq K(r-\theta)^{(1+\lambda) \beta-\alpha-1},
$$

and $(1+\lambda) \beta-\alpha>0$ since $\alpha<\frac{\lambda \beta+1}{2}<(1+\lambda) \beta$.

We need the following extension of the fractional derivative to the multiplicative functional $x \otimes y$, defined for $r \in[0, b)$ :

$$
\mathcal{D}_{b-}^{1-\alpha}(x \otimes y)(r)=\frac{(-1)^{1-\alpha}}{\Gamma(\alpha)}\left(\frac{(x \otimes y)_{r, b}}{(b-r)^{1-\alpha}}+(1-\alpha) \int_{r}^{b} \frac{(x \otimes y)_{r, s}}{(s-r)^{2-\alpha}} d s\right) .
$$

By Lemma 6.3 the function $\mathcal{D}_{b-}^{1-\alpha}(x \otimes y)(r)$ is Hölder continuous of order $\beta$. If $x$ and $y$ are continuously differentiable functions and the multiplicative functional $x \otimes y$ is given by (3.3), then

$$
\mathcal{D}_{b-}^{1-\alpha}(x \otimes y)(r)=\frac{(-1)^{1-\alpha}}{\Gamma(\alpha)} \int_{r<\xi<\eta<b}(\eta-r)^{\alpha-1} d x_{\xi} d y_{\eta} .
$$

We are now ready to define the integral $\int_{a}^{b} f\left(x_{r}\right) d y_{r}$.

Definition 3.2. Let $(x, y, x \otimes y)$ be in $M_{m, d}^{\beta}(0, T)$. Let $f: \mathbb{R}^{m} \rightarrow \mathbb{R}^{d}$ be a continuously differentiable function such that $f^{\prime}$ is locally $\lambda$-Hölder continuous, where $\lambda>\frac{1}{\beta}-2$. Fix $\alpha>0$ such that $1-\beta<\alpha<2 \beta$, and $\alpha<\frac{\lambda \beta+1}{2}$. Then, for any $0 \leq a<b \leq T$ we define

$$
\begin{aligned}
& \int_{a}^{b} f\left(x_{r}\right) d y_{r}=(-1)^{\alpha} \sum_{j=1}^{d} \int_{a}^{b} \widehat{D}_{a+}^{\alpha} f_{j}(x)(r) D_{b-}^{1-\alpha} y_{b-}^{j}(r) d r-(-1)^{2 \alpha-1} \\
& \times \sum_{i=1}^{m} \sum_{j=1}^{d} \int_{a}^{b} D_{a+}^{2 \alpha-1} \partial_{i} f_{j}(x)(r) D_{b-}^{1-\alpha} \mathcal{D}_{b-}^{1-\alpha}(x \otimes y)^{i, j}(r) d r .
\end{aligned}
$$

Notice that the fractional derivatives $D_{b-}^{1-\alpha} y_{b-}^{j}(r)$ and $D_{b-}^{1-\alpha} \mathcal{D}_{b-}^{1-\alpha}(x \otimes y)^{i, j}(r)$ are well defined because the functions $y^{j}$ and $\mathcal{D}_{b-}^{1-\alpha}(x \otimes y)^{i, j}$ are $\beta$-Hölder continuous.

The following theorem asserts that this definition is coherent with the classical notion of integral and will allow us to deduce estimates in the Hölder norm.

Theorem 3.3. Suppose $y:[0, T] \rightarrow \mathbb{R}^{d}$ is continuously differentiable and $x$ : $[0, T] \rightarrow \mathbb{R}^{m}$ is $\beta$-Hölder continuous. Let $x \otimes y$ be defined by $(x \otimes y)_{s, t}^{i, j}=$ $\int_{s}^{t}\left(x_{\xi}^{i}-x_{s}^{i}\right)\left(y^{j}\right)_{\xi}^{\prime} d \xi$. Assume that $f$ satisfies the assumptions of Definition 3.2 . Then, the integral $\int_{a}^{b} f\left(x_{r}\right) d y_{r}$ introduced in (3.11) coincides with

$$
\sum_{j=1}^{d} \int_{a}^{b} f_{j}\left(x_{r}\right)\left(y^{j}\right)_{r}^{\prime} d r
$$


Proof. To simplify the proof we take $m=d=1$. We have

$$
\begin{aligned}
\int_{a}^{b} f\left(x_{r}\right) y_{r}^{\prime} d r & =\int_{a}^{b}\left[f\left(x_{r}\right)-f^{\prime}\left(x_{r}\right)\left(x_{r}-x_{a}\right)\right] y_{r}^{\prime} d r+\int_{a}^{b} f^{\prime}\left(x_{r}\right)\left(x_{r}-x_{a}\right) y_{r}^{\prime} d r \\
& :=A_{1}+A_{2} .
\end{aligned}
$$

We can express the terms $A_{1}$ and $A_{2}$ using the fractional integration by parts formula (2.9). In this way we obtain

$$
A_{1}=(-1)^{\alpha} \int_{a}^{b} D_{a+}^{\alpha}\left[f(x .)-f^{\prime}(x .)\left(x .-x_{a}\right)\right](r) D_{b-}^{1-\alpha} y_{b-}(r) d r .
$$

Let us compute the first fractional derivative in the expression (3.12):

$$
\begin{aligned}
& D_{a+}^{\alpha}\left[f(x .)-f^{\prime}(x .)\left(x .-x_{a}\right)\right](r) \\
= & \frac{1}{\Gamma(1-\alpha)}\left(\frac{f\left(x_{r}\right)-f^{\prime}\left(x_{r}\right)\left(x_{r}-x_{a}\right)}{(r-a)^{\alpha}}\right. \\
& \left.+\alpha \int_{a}^{r} \frac{f\left(x_{r}\right)-f^{\prime}\left(x_{r}\right)\left(x_{r}-x_{a}\right)-\left[f\left(x_{\theta}\right)-f^{\prime}\left(x_{\theta}\right)\left(x_{\theta}-x_{a}\right)\right]}{(r-\theta)^{\alpha+1}} d \theta\right) \\
= & \widehat{D}_{a+}^{\alpha} f(x)(r)-\frac{\left(x_{r}-x_{a}\right)}{\Gamma(1-\alpha)}\left(\frac{f^{\prime}\left(x_{r}\right)}{(r-a)^{\alpha}}+\alpha \int_{a}^{r} \frac{f^{\prime}\left(x_{r}\right)-f^{\prime}\left(x_{\theta}\right)}{(r-\theta)^{\alpha+1}} d \theta\right) \\
= & \widehat{D}_{a+}^{\alpha} f(x)(r)-\left(x_{r}-x_{a}\right) D_{a+}^{\alpha} f^{\prime}(x)(r) .
\end{aligned}
$$

As a consequence, we obtain

$$
\begin{aligned}
A_{1}= & (-1)^{\alpha} \int_{a}^{b} \widehat{D}_{a+}^{\alpha} f(x)(r) D_{b-}^{1-\alpha} y_{b-}(r) d r \\
& -(-1)^{\alpha} \int_{a}^{b}\left(x_{r}-x_{a}\right) D_{a+}^{\alpha} f^{\prime}(x)(r) D_{b-}^{1-\alpha} y_{b-}(r) d r .
\end{aligned}
$$

On the other hand, applying again (2.9) yields

$$
\begin{aligned}
A_{2} & =\int_{a}^{b} f^{\prime}\left(x_{r}\right) d(x \otimes y)_{a, \cdot}(r) \\
& =(-1)^{\alpha} \int_{a}^{b} D_{a+}^{\alpha} f^{\prime}(x)(r) D_{b-}^{1-\alpha}(x \otimes y)_{a, \cdot, b-}(r) d r .
\end{aligned}
$$

We have, using (3.1),

$$
\begin{aligned}
& D_{b-}^{1-\alpha}(x \otimes y)_{a, \cdot, b-}(r) \\
= & \frac{(-1)^{1-\alpha}}{\Gamma(\alpha)}\left(\frac{(x \otimes y)_{a, r}-(x \otimes y)_{a, b}}{(b-r)^{1-\alpha}}+(1-\alpha) \int_{r}^{b} \frac{(x \otimes y)_{a, r}-(x \otimes y)_{a, \theta}}{(\theta-r)^{2-\alpha}} d \theta\right) \\
= & \frac{(-1)^{1-\alpha}}{\Gamma(\alpha)}\left(\frac{-(x \otimes y)_{r, b}-\left(x_{r}-x_{a}\right)\left(y_{b}-y_{r}\right)}{(b-r)^{1-\alpha}}\right. \\
& \left.+(1-\alpha) \int_{r}^{b} \frac{-(x \otimes y)_{r, \theta}-\left(x_{r}-x_{a}\right)\left(y_{\theta}-y_{r}\right)}{(\theta-r)^{2-\alpha}} d \theta\right) \\
= & -\mathcal{D}_{b-}^{1-\alpha}(x \otimes y)(r)+\left(x_{r}-x_{a}\right) D_{b-}^{1-\alpha} y_{b-}(r) .
\end{aligned}
$$


Substituting (3.15) into (3.14) and using (3.13) we get

$$
\begin{aligned}
\int_{a}^{b} f\left(x_{r}\right) d y_{r}= & (-1)^{\alpha} \int_{a}^{b} \widehat{D}_{a+}^{\alpha} f(x)(r) D_{b-}^{1-\alpha} y_{b-}(r) d r \\
& -(-1)^{\alpha} \int_{a}^{b} D_{a+}^{\alpha} f^{\prime}(x)(r) \mathcal{D}_{b-}^{1-\alpha}(x \otimes y)(r) d r .
\end{aligned}
$$

Finally, using the fractional integration by parts formula (2.8) we obtain

$$
\begin{aligned}
& \int_{a}^{b} D_{a+}^{\alpha} f^{\prime}(x)(r) \mathcal{D}_{b-}^{1-\alpha}(x \otimes y)(r) d r \\
= & (-1)^{\alpha-1} \int_{a}^{b} D_{a+}^{2 \alpha-1} f^{\prime}(x)(r) D_{b-}^{1-\alpha} \mathcal{D}_{b-}^{1-\alpha}(x \otimes y)(r) d r,
\end{aligned}
$$

which yields the desired result.

In the sequel, $k$ will denote a generic constant that may depend on the parameters $\beta, \alpha$, and $\lambda$. We have the following estimate.

Proposition 3.4. Let $(x, y, x \otimes y)$ be in $M_{m, d}^{\beta}(0, T)$. Assume that $f: \mathbb{R}^{m} \rightarrow \mathbb{R}^{d}$ is a continuously differentiable function such that $f^{\prime}$ is bounded and $\lambda$-Hölder continuous, where $\lambda>\frac{1}{\beta}-2$. Then for any $0 \leq a<b \leq T$ we have

$$
\begin{gathered}
\left\|\int f\left(x_{r}\right) d y_{r}\right\|_{a, b, \beta} \leq k\left|f\left(x_{a}\right)\right|\|y\|_{a, b, \beta}+k \Phi_{a, b, \beta}(x, y) \\
\times\left(\left\|f^{\prime}\right\|_{\infty}+\left\|f^{\prime}\right\|_{\lambda}\|x\|_{a, b, \beta}^{\lambda}(b-a)^{\lambda \beta}\right)(b-a)^{\beta},
\end{gathered}
$$

where $\Phi_{a, b, \beta}(x, y)$ is defined in (3.6).

Remark: In (3.16) we can replace $\left\|f^{\prime}\right\|_{\infty}$ by $\left\|f^{\prime}(x)\right\|_{a, b, \infty}$ and $\left\|f^{\prime}\right\|_{\lambda}$ by the $\lambda$-Hölder norm of $f^{\prime}$ on the interval $[\min x, \max x]$.

Proof. First we have, for any $r \in[a, b]$,

$$
\left|\widehat{D}_{a+}^{\alpha} f(x)(r)\right| \leq k\left(\left|f\left(x_{r}\right)\right|(r-a)^{-\alpha}+\left\|f^{\prime}\right\|_{\lambda}\|x\|_{a, r, \beta}^{1+\lambda}(r-a)^{(\lambda+1) \beta-\alpha}\right),
$$

and

$$
\left|D_{b-}^{1-\alpha} y_{b-}(r)\right| \leq k\|y\|_{r, b, \beta}(b-r)^{\alpha+\beta-1} .
$$

From (3.10) we get:

$$
\left|\mathcal{D}_{b-}^{1-\alpha}(x \otimes y)(r)\right| \leq k\|x \otimes y\|_{r, b, 2 \beta}(b-r)^{2 \beta+\alpha-1} .
$$

Using (3.10), (6.3) and (3.19) yields

$$
\left|D_{b-}^{1-\alpha} \mathcal{D}_{b-}^{1-\alpha}(x \otimes y)(r)\right| \leq k \Phi_{r, b, \beta}(x, y)(b-r)^{2 \beta+2 \alpha-2} .
$$

Moreover,

$$
\left|D_{a+}^{2 \alpha-1} f^{\prime}(x)(r)\right| \leq k\left(\left|f^{\prime}\left(x_{r}\right)\right|(r-a)^{1-2 \alpha}+\left\|f^{\prime}\right\|_{\lambda}\|x\|_{a, r, \beta}^{\lambda}(r-a)^{\lambda \beta-2 \alpha+1}\right) .
$$


Thus, using (3.11) and the inequalities (3.17), (3.18), (3.20), and (3.21) yields

$$
\begin{aligned}
\left|\int_{a}^{b} f\left(x_{r}\right) d y_{r}\right| & \leq k\|y\|_{a, b, \beta}\left(\int_{a}^{b}\left|f\left(x_{r}\right)\right|(r-a)^{-\alpha}(b-r)^{\alpha+\beta-1} d r\right. \\
+ & \left.\left\|f^{\prime}\right\|_{\lambda}\|x\|_{a, b, \beta}^{1+\lambda} \int_{a}^{b}(r-a)^{(1+\lambda) \beta-\alpha}(b-r)^{\alpha+\beta-1} d r\right) \\
+ & k \int_{a}^{b}\left(\frac{\left|f^{\prime}\left(x_{r}\right)\right|}{(r-a)^{2 \alpha-1}}+\left\|f^{\prime}\right\|_{\lambda}\|x\|_{a, b, \beta}^{\lambda}(r-a)^{\lambda \beta-2 \alpha+1}\right) \\
& \times \Phi_{a, b, \beta}(x, y)(b-r)^{2 \beta+2 \alpha-2} d r .
\end{aligned}
$$

Therefore, we obtain

$$
\begin{aligned}
& \left|\int_{a}^{b} \quad f\left(x_{r}\right) d y_{r}\right| \leq k\left|f\left(x_{a}\right)\right|\|y\|_{a, b, \beta}(b-a)^{\beta} \\
& \quad+k\|y\|_{a, b, \beta}\|x\|_{a, b, \beta}\left\|f^{\prime}\right\|_{\infty}(b-a)^{2 \beta} \\
& \quad+k\|y\|_{a, b, \beta}\left\|f^{\prime}\right\|_{\lambda}\|x\|_{a, b, \beta}^{1+\lambda}(b-a)^{(\lambda+2) \beta} \\
& \quad+k \Phi_{a, b, \beta}(x, y)\left\|f^{\prime}\right\|_{\infty}(b-a)^{2 \beta} \\
& \quad+k\left\|f^{\prime}\right\|_{\lambda}\|x\|_{a, b, \beta}^{\lambda} \Phi_{a, b, \beta}(x, y)(b-a)^{(\lambda+2) \beta}
\end{aligned}
$$

and this implies (3.16) easily.

The estimate (3.16) implies that for a fixed $x$, the mapping $(y, x \otimes y) \rightarrow \int f\left(x_{r}\right) d y_{r}$ is continuous with respect to the $\beta$-norm. That is, if $\left(x, y^{n}, x \otimes y^{n}\right)$ is a sequence of $\beta$-Hölder continuous multiplicative functionals such that

$$
\begin{aligned}
\left\|y-y^{n}\right\|_{\beta} & \rightarrow 0, \\
\left\|x \otimes y-x \otimes y^{n}\right\|_{2 \beta} & \rightarrow 0,
\end{aligned}
$$

as $n$ tends to infinity, then

$$
\left\|\int f\left(x_{r}\right) d y_{r}-\int f\left(x_{r}\right) d y_{r}^{n}\right\|_{\beta} \rightarrow 0 .
$$

Suppose that $(x, y, x \otimes y)$ belongs to $M_{m, d}^{\beta}(0, T)$. Then, the integral $\int f\left(x_{r}\right) d y_{r}$ introduced in Definition 3.2 does not depend on the parameter $\alpha$, and it coincides with the limit of the classical integrals $\int f\left(x_{r}\right) d y_{r}^{n}$ when $y^{n}$ is a sequence of piecewise continuously differentiable functions such that (3.22) holds and $(x \otimes y)_{s, t}^{i, j}=$ $\int_{s}^{t}\left(x_{\xi}^{i}-x_{s}^{i}\right)\left(y^{j}\right)_{\xi}^{\prime} d \xi$ satisfies (3.23).

Set $t_{i}^{n}=\frac{i T}{n}$ for $i=0,1, \ldots, n$. If $y$ is $\beta$-Hölder continuous, then the sequence of functions

$$
y_{t}^{n}=y_{0} \mathbf{1}_{\{0\}}(t)+\sum_{i=1}^{n} \mathbf{1}_{\left(t_{i-1}^{n}, t_{i}^{n}\right]}(t)\left[y_{t_{i-1}^{n}}+\frac{n}{T}\left(t-t_{i-1}^{n}\right)\left(y_{t_{i}^{n}}-y_{t_{i-1}^{n}}\right)\right]
$$

converges to $y$ in the $\beta^{\prime}$-norm for any $\beta^{\prime}<\beta$. Fix $\frac{1}{3}<\beta^{\prime}<\beta$ and assume that $\int_{s}^{t}\left(x_{r}-x_{s}\right) d y_{r}^{n}$ converges in the $\beta^{\prime}$-norm to $(x \otimes y)_{s, t}$ as $n$ tends to infinity. Then 
(3.24) holds with $\beta=\beta^{\prime}$. In particular, this means that, under these assumptions, we have

$$
\int_{0}^{T} f\left(x_{r}\right) d y_{r}=\lim _{n \rightarrow \infty} \sum_{i=1}^{n} \frac{n}{T}\left(\int_{t_{i-1}^{n}}^{t_{i}^{n}} f\left(x_{s}\right) d s\right)\left(y_{t_{i}^{n}}-y_{t_{i-1}^{n}}\right) .
$$

For any $p \geq 1$, the $p$ variation of a function $x:[0, T] \rightarrow \mathbb{R}$ is defined as

$$
\operatorname{Var}_{p}(x)=\sup _{\pi}\left(\sum_{i=1}^{n}\left|x\left(t_{i}^{n}\right)-x\left(t_{i-1}^{n}\right)\right|^{p}\right)^{1 / p},
$$

where $\pi=\left\{0=t_{0}<\cdots<t_{n}=T\right\}$ runs over all partitions of $[0, T]$. Notice that

$$
\operatorname{Var}_{1 / \beta}(x) \leq\|x\|_{\beta} .
$$

Then, for any $(x, y, x \otimes y)$ in $M_{m, d}^{\beta}(0, T)$ and any function $f$ satisfying the hypotheses of Definition 3.2, the integral $\int_{0}^{T} f\left(x_{r}\right) d y_{r}$ coincides with the integral defined using the $\frac{1}{\beta}$-variation norm (see [13]). This implies that $\int_{0}^{T} f\left(x_{s}\right) d y_{s}$ is given by the limit of Riemann sums of the form

$$
\int_{0}^{T} f\left(x_{s}\right) d y_{s}=\lim _{|\pi| \rightarrow 0} \sum_{i=1}^{n}\left[f\left(x_{t_{i-1}}\right)\left(y_{t_{i}}-y_{t_{i-1}}\right)+f^{\prime}\left(x_{t_{i-1}}\right)(x \otimes y)_{t_{i-1}, t_{i}}\right],
$$

where $\pi=\left\{0=t_{0}<\cdots<t_{n}=T\right\}$ runs over all partitions of $[0, T]$.

The following proposition compares the integrals defined using two different multiplicative functionals.

Proposition 3.5. Let $(x, y, x \otimes y)$ and $(x, y, x \widehat{\otimes} y)$ be two elements in $M_{m, d}^{\beta}(0, T)$. Set $\varphi(s, t)=\left(\varphi^{i, j}(s, t)\right)_{1 \leq i \leq m, 1 \leq j \leq d}$, where

$$
\varphi^{i, j}(s, t)=\left(x^{i} \otimes y^{j}\right)_{s, t}-\left(x^{i} \widehat{\otimes} y^{j}\right)_{s, t},
$$

and $\varphi(t)=\varphi(0, t)$. Then $\varphi(s, t)=\varphi(t)-\varphi(s)$ and the function $\varphi:[0, T] \rightarrow \mathbb{R}^{m} \otimes \mathbb{R}^{d}$ is $2 \beta$-Hölder continuous. Suppose that $f: \mathbb{R}^{m} \rightarrow \mathbb{R}^{d}$ is a continuously differentiable function such that $f^{\prime}$ is locally $\lambda$-Hölder continuous, where $\lambda>\frac{1}{\beta}-2$. Fix $\alpha>0$ such that $1-\beta<\alpha<2 \beta$, and $\alpha<\frac{\lambda \beta+1}{2}$. Then, for any $0 \leq a<b \leq T$ we have

$$
\int_{a}^{b} f\left(x_{r}\right) d y_{r}-\int_{a}^{b} f\left(x_{r}\right) \widehat{d} y_{r}=\sum_{i=1}^{m} \sum_{j=1}^{d} \int_{a}^{b} \partial_{i} f_{j}\left(x_{r}\right) d \varphi^{i, j}(r) .
$$

Notice that the integral on the right-hand side of (3.25) exists as a RiemannStieltjes integral because $\partial_{i} f_{j}\left(x_{r}\right)$ is $\lambda \beta$-Hölder continuous, $\varphi^{i, j}$ is $2 \beta$-Hölder continuous, and $\lambda \beta+2 \beta>1$.

Proof. To simplify we assume $m=d=1$. From (3.11) we have

$$
\int_{a}^{b} f\left(x_{r}\right) d y_{r}-\int_{a}^{b} f\left(x_{r}\right) \widehat{d} y_{r}=-(-1)^{2 \alpha-1} \int_{a}^{b} D_{a+}^{2 \alpha-1} f^{\prime}(x)(r) D_{b-}^{1-\alpha} \mathcal{D}_{b-}^{1-\alpha} \varphi(r) d r .
$$

The definition of the operator $\mathcal{D}_{b-}^{1-\alpha}$ yields

$$
\begin{aligned}
\mathcal{D}_{b-}^{1-\alpha} \varphi(r) & =\frac{(-1)^{1-\alpha}}{\Gamma(\alpha)}\left(\frac{\varphi(b)-\varphi(r)}{(b-r)^{1-\alpha}}+(1-\alpha) \int_{r}^{b} \frac{\varphi(s)-\varphi(r)}{(s-r)^{2-\alpha}} d s\right) \\
& =-D_{b-}^{1-\alpha} \varphi_{b-}(r) .
\end{aligned}
$$


As a consequence, using (2.3) and (2.9) yields

$$
\begin{aligned}
\int_{a}^{b} f\left(x_{r}\right) d y_{r}-\int_{a}^{b} f\left(x_{r}\right) \widehat{d} y_{r} & =(-1)^{2 \alpha-1} \int_{a}^{b} D_{a+}^{2 \alpha-1} f^{\prime}(x)(r) D_{b-}^{1-\alpha} D_{b-}^{1-\alpha} \varphi_{b-}(r) d r \\
& =(-1)^{2 \alpha-1} \int_{a}^{b} D_{a+}^{2 \alpha-1} f^{\prime}(x)(r) D_{b-}^{2-2 \alpha} \varphi_{b-}(r) d r \\
& =\int_{a}^{b} f^{\prime}\left(x_{r}\right) d \varphi(r)
\end{aligned}
$$

In order to handle differential equations we need to introduce the tensor product of two multiplicative functionals:

Definition 3.6. Suppose that $(x, y, x \otimes y)$ and $(y, z, y \otimes z)$ belong to $M_{1,1}^{\beta}(0, T)$. Then, for all $a \leq b \leq c$, we define

$$
\begin{aligned}
& \left(x \otimes(y \otimes z)_{, c}\right)_{a, b} \\
= & \frac{(-1)^{\alpha}}{\Gamma(1-\alpha)} \int_{a}^{b}\left(\frac{\left(x_{r}-x_{a}\right)\left(z_{c}-z_{r}\right)}{(r-a)^{\alpha}}+\alpha \int_{a}^{r} \frac{\left(z_{r}-z_{\theta}\right)\left(x_{\theta}-x_{r}\right)}{(r-\theta)^{\alpha+1}} d \theta\right) \\
& \times D_{b-}^{1-\alpha} y_{b-}(r) d r \\
& -\frac{(-1)^{2 \alpha-1}}{\Gamma(2-2 \alpha)} \int_{a}^{b}\left[\frac{z_{c}-z_{r}}{(r-a)^{2 \alpha-1}}+(2 \alpha-1) \int_{a}^{r} \frac{z_{\theta}-z_{r}}{(r-\theta)^{2 \alpha}} d \theta\right] \\
& \quad \times D_{b-}^{1-\alpha} \mathcal{D}_{b-}^{1-\alpha}(x \otimes y)(r) d r \\
+ & \frac{(-1)^{2 \alpha-1}}{\Gamma(2-2 \alpha)} \int_{a}^{b}\left[\frac{x_{r}-x_{a}}{(r-a)^{2 \alpha-1}}+(2 \alpha-1) \int_{a}^{r} \frac{x_{r}-x_{\theta}}{(r-\theta)^{2 \alpha}} d \theta\right] \\
& \quad \times D_{b-}^{1-\alpha} \mathcal{D}_{b-}^{1-\alpha}(y \otimes z)(r) d r .
\end{aligned}
$$

We have the following result.

Proposition 3.7. If the function $y$ is continuously differentiable and for all $a \leq b$,

$$
\begin{aligned}
& (y \otimes z)_{a, b}=\int_{a}^{b}\left(z_{b}-z_{r}\right) y_{r}^{\prime} d r, \\
& (x \otimes y)_{a, b}=\int_{a}^{b}\left(x_{r}-x_{a}\right) y_{r}^{\prime} d r,
\end{aligned}
$$

then

$$
\left(x \otimes(y \otimes z)_{,, c}\right)_{a, b}=\int_{a}^{b}\left(x_{r}-x_{a}\right)\left(z_{c}-z_{r}\right) y_{r}^{\prime} d r .
$$

Proof. It suffices to apply formula (3.11) with $m=2, d=1, f(x, z)=x z$ and the functions $x_{t}-x_{a}$ and $z_{c}-z_{t}$.

Then, the following estimate follows from Definition 3.6 and the inequalities (3.18) and (3.20).

Proposition 3.8. Suppose that $(x, y, x \otimes y)$ and $(y, z, y \otimes z)$ belong to $M_{1,1}^{\beta}(0, T)$. Then, for any $a \leq b$ we have

$$
\begin{aligned}
\left|\left(x \otimes(y \otimes z)_{\cdot, c}\right)_{a, b}\right| \leq & k \Phi_{a, b, \beta}(x, y)\|z\|_{a, b, \beta}(b-a)^{2 \beta}(c-a)^{\beta} \\
& +k\|x\|_{a, b, \beta}\|y \otimes z\|_{a, b, 2 \beta}(b-a)^{3 \beta}
\end{aligned}
$$


If $b=c$ we write $\left(x \otimes(y \otimes z)_{\cdot, b}\right)_{a, b}=(x \otimes y \otimes z)_{a, b}$. Notice that if the functions $x, y$ and $z$ are continuously differentiable, then

$$
(x \otimes y \otimes z)_{a, b}=\int_{a<r<\theta<\sigma<b} x_{r}^{\prime} y_{\theta}^{\prime} z_{\sigma}^{\prime} d r d \theta d \sigma .
$$

Proposition 3.8 implies

$$
\|x \otimes y \otimes z\|_{a, b, 3 \beta} \leq k \Phi_{a, b, \beta}(x, y, z),
$$

where $\Phi_{a, b, \beta}(x, y, z)$ is defined in (3.7).

Proposition 3.8 also implies that $\left(x,(y \otimes z)_{,, c},\left(x \otimes(y \otimes z)_{,, c}\right)\right)$ is a $\beta$-Hölder continuous functional on the interval $[0, c]$. As a consequence, if $f$ satisfies the assumptions of Definition 3.2, we can define the integral $\int_{a}^{b} f\left(x_{r}\right) d_{r}(y \otimes z)_{r, c}$, for all $a \leq b \leq c$. The following estimate for this integral will be needed to solve differential equations.

Proposition 3.9. Suppose that $(x, y, x \otimes y)$ and $(y, z, y \otimes z)$ belong to $M_{m, d}^{\beta}(0, T)$. Let $f: \mathbb{R}^{m} \rightarrow \mathbb{R}^{d}$ be a continuously differentiable function such that $f^{\prime}$ is $\lambda$-Hölder continuous and bounded, where $\lambda>\frac{1}{\beta}-2$. Fix $\alpha>0$ such that $1-\beta<\alpha<2 \beta$, $\alpha<\frac{\lambda \beta+1}{2}$. Then the following estimate holds:

$$
\begin{aligned}
\left|\int_{a}^{b} f\left(x_{r}\right) d_{r}(y \otimes z)_{r, b}\right| \leq & k\left|f\left(x_{a}\right)\right| \Phi_{a, b, \beta}(y, z)(b-a)^{2 \beta} \\
+ & k\left(\left\|f^{\prime}\right\|_{\infty}+\left\|f^{\prime}\right\|_{\lambda}\|x\|_{a, b, \beta}^{\lambda}(b-a)^{\lambda \beta}\right) \\
& \times \Phi_{a, b, \beta}(x, y, z)(b-a)^{3 \beta} .
\end{aligned}
$$

Proof. To simplify the proof we will assume $d=m=1$. From (3.1) it is easy to see that

$$
\left\|(x \otimes y)_{\cdot, b}\right\|_{a, b, \beta} \leq \Phi_{a, b, \beta}(x, y)(b-a)^{\beta},
$$

and from Proposition 3.8 we have

$$
\left\|x \otimes(y \otimes z)_{,, b}\right\|_{a, b, 2 \beta} \leq k \Phi_{a, b, \beta}(x, y, z)(b-a)^{\beta} .
$$

From (3.16), (3.29), and (3.30) we obtain

$$
\begin{aligned}
& \left|\int_{a}^{b} f\left(x_{r}\right) d_{r}(y \otimes z)_{r, b}\right| \\
\leq \quad & k\left|f\left(x_{a}\right)\right|\left\|(y \otimes z)_{\cdot, b}\right\|_{a, b, \beta}(b-a)^{\beta} \\
& +k\left[\Phi_{a, b, \beta}\left(x,(y \otimes z)_{\cdot, b}\right)\left(\left\|f^{\prime}\right\|_{\infty}+\left\|f^{\prime}\right\|_{\lambda}\|x\|_{a, b, \beta}^{\lambda}(b-a)^{\lambda \beta}\right)\right](b-a)^{2 \beta} \\
\leq \quad & k\left|f\left(x_{a}\right)\right| \Phi_{a, b, \beta}(y, z)(b-a)^{2 \beta} \\
& +k\left[\Phi_{a, b, \beta}(x, y, z)\left(\left\|f^{\prime}\right\|_{\infty}+\left\|f^{\prime}\right\|_{\lambda}\|x\|_{a, b, \beta}^{\lambda}(b-a)^{\lambda \beta}\right)\right](b-a)^{3 \beta},
\end{aligned}
$$

which implies the desired result. 


\section{Differential EQUATIONS DRIVEn By ROUGH PATHS}

Suppose that $(y, y, y \otimes y)$ belongs to $M_{d, d}^{\beta}(0, T)$. We aim to solve the differential equation

$$
x_{t}=x_{0}+\int_{0}^{t} f\left(x_{r}\right) d y_{r},
$$

where $f: \mathbb{R}^{m} \rightarrow \mathbb{R}^{m} \otimes \mathbb{R}^{d}$. The main idea is to consider the enlarged unknown $(x, x \otimes y)$, where

$$
(x \otimes y)_{s, t}^{k, l}=\sum_{j=1}^{d} \int_{s}^{t} f_{j}^{k}\left(x_{r}\right) d(y \otimes y)_{\cdot, t}^{j, l}(r) .
$$

By formula (3.11), the right-hand side of Equation (4.1) is a function of $(x, y, x \otimes y)$, and the right-hand side of Equation (4.2) is a function of $(x, y \otimes y, x \otimes(y \otimes y))$. On the other hand, by Equation (3.26),$x \otimes(y \otimes y)$ is a functional of $(x, y, x \otimes y, y \otimes y)$.

By definition, a solution of Equation (4.1) is an element of $M_{m, d}^{\beta}(0, T)$ such that (4.1) and (4.2) hold. This is equivalent to saying that the following system of equations holds

$$
\begin{aligned}
x_{t}= & x_{0}+(-1)^{\alpha} \sum_{j=1}^{d} \int_{0}^{t} \widehat{D}_{0+}^{\alpha} f_{j}(x)(s) D_{t-}^{1-\alpha} y_{t-}^{j}(s) d s \\
& -(-1)^{2 \alpha-1} \sum_{i=1}^{m} \sum_{j=1}^{d} \int_{0}^{t} D_{0+}^{2 \alpha-1} \partial_{i} f_{j}(x)(s) \\
& \times D_{t-}^{1-\alpha} \mathcal{D}_{t-}^{1-\alpha}(x \otimes y)^{i, j}(s) d s \\
(x \otimes y)_{s, t}^{k, l}= & (-1)^{\alpha} \sum_{i=1}^{d} \int_{s}^{t} \widehat{D}_{s+}^{\alpha} f_{i}^{k}(x)(r) D_{t-}^{1-\alpha}(y \otimes y)_{\cdot, t-}^{i, l}(r) d r \\
& -(-1)^{2 \alpha-1} \sum_{i=1}^{m} \sum_{j=1}^{d} \int_{s}^{t} D_{s+}^{2 \alpha-1} \partial_{i} f_{j}(x)(r) \\
& \times D_{t-}^{1-\alpha} \mathcal{D}_{t-}^{1-\alpha}\left(x \otimes(y \otimes y)_{\cdot, t}\right)^{k, i, l}(r) d r
\end{aligned}
$$

and

$$
\begin{aligned}
& \left(x \otimes(y \otimes y)_{\cdot, t}\right)_{s, t}^{i, j, k} \\
= & \frac{(-1)^{\alpha}}{\Gamma(1-\alpha)} \int_{s}^{t}\left(\frac{\left(x_{r}^{i}-x_{s}^{i}\right)\left(y_{t}^{k}-y_{r}^{k}\right)}{(r-s)^{\alpha}}+\alpha \int_{s}^{r} \frac{\left(y_{r}^{k}-y_{\theta}^{k}\right)\left(x_{\theta}^{i}-x_{r}^{i}\right)}{(r-\theta)^{\alpha+1}} d \theta\right) \\
& \times D_{t-}^{1-\alpha} y_{t-}^{j}(r) d r \\
& -\frac{(-1)^{2 \alpha-1}}{\Gamma(2-2 \alpha)} \int_{s}^{t}\left[\frac{y_{t}^{k}-y_{r}^{k}}{(r-s)^{2 \alpha-1}}+(2 \alpha-1) \int_{s}^{r} \frac{y_{\theta}^{k}-y_{r}^{k}}{(r-\theta)^{2 \alpha}} d \theta\right] \\
& +\frac{(-1)^{2 \alpha-1}}{\Gamma(2-2 \alpha)} \int_{s}^{t}\left[\frac{x_{r}^{i}-x_{a}^{i}}{(r-s)^{2 \alpha-1}}+(2 \alpha-1) \int_{s}^{r} \frac{x_{r}^{i}-x_{\theta}^{i}}{(r-\theta)^{2 \alpha}} d \theta\right] \\
& \quad \times D_{t-}^{1-\alpha} \mathcal{D}_{t-}^{1-\alpha}(y \otimes y)^{j, k}(r) d r .
\end{aligned}
$$


First we establish the existence of a solution.

Theorem 4.1. Let $(y, y, y \otimes y)$ be in $M_{d, d}^{\beta}(0, T)$. Let $f: \mathbb{R}^{m} \rightarrow \mathbb{R}^{m d}$ be a continuously differentiable function such that $f^{\prime}$ is $\lambda$-Hölder continuous, where $\lambda>\frac{1}{\beta}-2$, and $f$ and $f^{\prime}$ are bounded. Set

$$
\rho_{f}=\|f\|_{\infty}+\left\|f^{\prime}\right\|_{\infty}+\left\|f^{\prime}\right\|_{\lambda} .
$$

Then there is a solution $(x, y, x \otimes y)$ in $M_{m, d}^{\beta}(0, T)$ to Equations (4.1)-(4.2). Moreover, the function $x$ satisfies the estimate

$$
\sup _{0 \leq t \leq T}\left|x_{t}\right| \leq\left|x_{0}\right|+1+T\left\{2 k \rho_{f}\left[\|y\|_{\beta}+\frac{\|y \otimes y\|_{2 \beta}}{\|y\|_{\beta}}\right]\right\}^{1 / \beta}
$$

where $k$ is a universal constant depending only on $\alpha, \beta$ and $\lambda$, with the convention $\frac{\|y \otimes y\|_{2 \beta}}{\|y\|_{\beta}}=0$ if $\|y\|_{\beta}=0$.

Proof. To simplify the proof we will assume $d=m=1$. The proof will be done in several steps.

Step 1. Fix $\alpha>0$ such that $1-\beta<\alpha<2 \beta, \alpha<\frac{\lambda \beta+1}{2}$.

Consider the mapping $J: M_{1,1}^{\beta}(0, T) \rightarrow M_{1,1}^{\beta}(0, T)$ given by $J(x, y, x \otimes y)=$ $\left(J_{1}, y, J_{2}\right)$, where $J_{1}$ and $J_{2}$ are the right-hand sides of equations (4.1) and (4.2), respectively. This mapping is well defined because for each $(x, y, x \otimes y)$ in $M_{1,1}^{\beta}(0, T)$, $\left(J_{1}, y, J_{2}\right)$ is a real-valued $\beta$-Hölder continuous multiplicative functional. We need some a priori estimates of the Hölder norms of $J_{1}$ and $J_{2}$ in terms of the Hölder norms of $x, y$ and $x \otimes y$. From (3.16) and (3.28) it follows that

$$
\begin{aligned}
\left\|J_{1}\right\|_{s, t, \beta} \leq & k\left[\left|f\left(x_{s}\right)\right|\|y\|_{s, t, \beta}+\Phi_{s, t, \beta}(x, y)\right. \\
& \left.\times\left(\left\|f^{\prime}(x)\right\|_{s, t, \infty}+\left\|f^{\prime}\right\|_{\lambda}\|x\|_{s, t, \beta}^{\lambda}(t-s)^{\lambda \beta}\right)(t-s)^{\beta}\right]
\end{aligned}
$$

and

$$
\begin{aligned}
\left\|J_{2}\right\|_{s, t, 2 \beta} \leq & k\left|f\left(x_{s}\right)\right| \Phi_{s, t, \beta}(y, y) \\
& +k\left(\left\|f^{\prime}\right\|_{\infty}+\left\|f^{\prime}\right\|_{\lambda}\|x\|_{s, t, \beta}^{\lambda}(t-s)^{\lambda \beta}\right) \\
& \times \Phi_{s, t, \beta}(x, y, y)(t-s)^{\beta} .
\end{aligned}
$$

Step 2. Set

$$
\alpha(y):=\left(2 k \rho_{f}\left[\|y\|_{\beta}+\frac{\|y \otimes y\|_{2 \beta}}{\|y\|_{\beta}}\right]\right)^{1 / \beta},
$$

where $k$ is the constant appearing in formulas (4.7) and (4.8). Fix $s, t$ such that

$$
0<t-s \leq \frac{1}{\alpha(y)}
$$

Notice that

$$
(t-s)^{\beta} \leq \alpha(y)^{-\beta} \leq\left(2 k \rho_{f}\|y\|_{\beta}\right)^{-1} .
$$

Let $C_{s, t}^{y}$ be the set of elements $(x, y, x \otimes y) \in M_{1,1}^{\beta}(0, T)$ such that

$$
\begin{aligned}
\|x\|_{s, t, \beta} & \leq 2 k \rho_{f}\|y\|_{\beta}, \\
(t-s)^{\beta}\|x \otimes y\|_{s, t, 2 \beta} & \leq\|y\|_{\beta} .
\end{aligned}
$$


We claim that $J\left(C_{s, t}^{y}\right) \subset C_{s, t}^{y}$. In fact, suppose that $(x, y, x \otimes y) \in C_{s, t}^{y}$. Then, (4.10) and (4.11) imply

$$
(t-s)^{\beta}\|x\|_{s, t, \beta} \leq 1 .
$$

The inequalities (4.12) and (4.13) imply

$$
(t-s)^{\beta} \Phi_{s, t, \beta}(x, y)=(t-s)^{\beta}\left(\|x \otimes y\|_{s, t, 2 \beta}+\|x\|_{s, t, \beta}\|y\|_{s, t, \beta}\right) \leq 2\|y\|_{\beta}
$$

and

$$
\begin{aligned}
(t-s)^{\beta} \Phi_{s, t, \beta}(x, y, y)= & (t-s)^{\beta}\left(\|x\|_{s, t, \beta}\|y\|_{s, t, \beta}^{2}\right. \\
& \left.+\|x\|_{s, t, \beta}\|y \otimes y\|_{s, t, 2 \beta}+\|y\|_{s, t, \beta}\|x \otimes y\|_{s, t, 2 \beta}\right) \\
\leq & 2\|y\|_{s, t, \beta}^{2}+\|y \otimes y\|_{s, t, 2 \beta} .
\end{aligned}
$$

From (4.7), (4.13) and (4.14) we obtain

$$
\left\|J_{1}\right\|_{s, t, \beta} \leq 2 k \rho_{f}\|y\|_{\beta},
$$

and from (4.8), (4.13), (4.15) and (4.9) we get

$$
\begin{aligned}
\left\|J_{2}\right\|_{s, t, 2 \beta} & \leq k\left[\|f\|_{\infty} \Phi_{s, t, \beta}(y, y)+\left(\left\|f^{\prime}\right\|_{\infty}+\left\|f^{\prime}\right\|_{\lambda}\right)\left(2\|y\|_{\beta}^{2}+\|y \otimes y\|_{2 \beta}\right)\right] \\
& \leq 2 k \rho_{f}\left(\|y\|_{\beta}^{2}+\|y \otimes y\|_{2 \beta}\right)=\alpha(y)^{\beta}\|y\|_{\beta} \\
& \leq(t-s)^{-\beta}\|y\|_{\beta} .
\end{aligned}
$$

Hence, $\left(J_{1}, y, J_{2}\right) \in C_{s, t}^{y}$ and the claim is proved.

Step 3. We can now proceed with the proof of the existence. Let $N$ be a natural number such that $\frac{T}{N}=\delta \leq \frac{1}{\alpha(y)}$. We partition the interval $[0, T]$ into $N$ subintervals of the same length and set $t_{i}=\frac{i T}{N}, i=0,1, \ldots, N-1$. We will make use of the notation $\|x\|_{i}=\|x\|_{t_{i-1}, t_{i}, \beta}$ and $\|x \otimes y\|_{i}=\|x \otimes y\|_{t_{i-1}, t_{i}, 2 \beta}$, for $i=1, \ldots, N-1$. From Step 2 we know that if that $x$ and $x \otimes y$ satisfy

$$
\begin{aligned}
\|x\|_{i} & \leq 2 k \rho_{f}\|y\|_{\beta}, \\
\|x \otimes y\|_{i} & \leq\|y\|_{\beta} \delta^{-\beta},
\end{aligned}
$$

for any $i=1, \ldots, N-1$, then the same inequalities hold for $J_{1}$ and $J_{2}$, that is,

$$
\begin{aligned}
&\left\|J_{1}\right\|_{i} \leq 2 k \rho_{f}\|y\|_{\beta}, \\
&\left\|J_{2}\right\|_{i} \leq\|y\|_{\beta} \delta^{-\beta} .
\end{aligned}
$$

Consequently, there is a constant $C_{1}$ such that

$$
\left\|J_{1}^{n}\right\|_{\beta}+\left\|J_{2}^{n}\right\|_{2 \beta} \leq C_{1} .
$$

This implies that the sequence of functions $J_{1}^{n}$ is equicontinuous and bounded in $C^{\beta}(0, T)$. Therefore, there exists a subsequence which converges in the $\beta^{\prime}$-Hölder norm if $\beta^{\prime}<\beta$. In the same way, there is a subsequence of $J_{2}^{n}$ which converges in the $\beta^{\prime}$-Hölder norm. The limit defines a $\beta$-Hölder continuous multiplicative functional $(x, y, x \otimes y)$. Using the continuity of the solution in this norm it is not difficult to show that the limit is a solution. This implies the existence of a solution, which satisfies (4.11) and (4.12) if (4.9) holds. 
Step 4. Let us now prove the estimate (4.6). By step 2, the solution we have constructed satisfies the estimates (4.11) and (4.12) if (4.9) holds. Then it follows that for any $r \in[s, t]$,

$$
\sup _{r \in[s, t]}\left|x_{r}\right| \leq\left|x_{s}\right|+(t-s)^{\beta}\|x\|_{s, t, \beta} \leq\left|x_{s}\right|+1 .
$$

Since the interval $[0, T]$ can be divided into $[T \alpha(y)]+1$ intervals of length $\frac{1}{\alpha(y)}$, the inequality (4.6) follows.

The following result provides the uniqueness of a solution under stronger hypotheses; moreover it provides the continuity of the solution in the input parameters $x_{0}, y$ and $y \otimes y$. For any vector $\bar{y}$ in $\mathbb{R}^{2 N}$, we set $\bar{y}=(y, \widetilde{y})$, where $y$ (resp. $\widetilde{y}$ ) is the vector formed with the first (resp. last) $N$ components of $\bar{y}$.

Theorem 4.2. Let $(y, y, y \otimes y)$ be an element of $M_{d, d}^{\beta}(0, T)$. Let $f: \mathbb{R}^{m} \rightarrow \mathbb{R}^{m d}$ be a twice continuously differentiable function such that $f^{\prime \prime}$ is $\lambda$-Hölder continuous, where $\lambda>\frac{1}{\beta}-2$, and $f, f^{\prime}$ and $f^{\prime \prime}$ are bounded. Then there is a unique solution $(x, y, x \otimes y) \in M_{m, d}^{\beta}(0, T)$ to Equations (4.1)-(4.2).

Suppose that $\bar{y}$ belongs to $M_{2 d, 2 d}^{\beta}(0, T)$, and set $\bar{y}=(y, \widetilde{y})$, where $y$ and $\widetilde{y}$ are $d$-dimensional vectors. Let $(\bar{x}, \bar{y}, \bar{x} \otimes \bar{y}) \in M_{2 m, 2 d}^{\beta}(0, T)$ be the unique solution to the Equation $\bar{x}_{t}=\bar{x}_{0}+\int_{0}^{t} \bar{f}\left(\bar{x}_{r}\right) d \bar{y}_{r}$, where $\bar{f}: \mathbb{R}^{2 m} \rightarrow \mathbb{R}^{2 d}$ is defined by $\bar{f}(\bar{x})=$ $(f(x), f(\widetilde{x}))$. Then

$\sup _{0 \leq t \leq T}\left|x_{t}-\widetilde{x}_{t}\right| \leq C\left\{\left|x_{0}-\widetilde{x}_{0}\right|+\|y-\widetilde{y}\|_{\beta}+\|(y-\widetilde{y}) \otimes y\|_{2 \beta}+\|\widetilde{y} \otimes(y-\widetilde{y})\|_{2 \beta}\right\}$,

where $C$ depends on $\|y\|_{\beta},\|\widetilde{y}\|_{\beta},\|y \otimes y\|_{2 \beta}, \beta, \lambda, T$, and $\widehat{\rho}_{f}$, and where

$$
\widehat{\rho}_{f}=\|f\|_{\infty}+\left\|f^{\prime}\right\|_{\infty}+\left\|f^{\prime}\right\|_{\lambda}+\left\|f^{\prime \prime}\right\|_{\infty}+\left\|f^{\prime \prime}\right\|_{\lambda} .
$$

Proof. To simplify the proof we will assume $d=m=1$. Notice that uniqueness follows from the estimate (4.18). So it suffices to show this inequality. To simplify, assume $\|y\|_{\beta}>0$ and $\|\widetilde{y}\|_{\beta}>0$. We fix $s<t$ such that $t-s \leq \frac{1}{\beta(y)} \wedge 1$, where $\beta$ is defined as follows:

$$
\beta(y)=\left(k \widehat{\rho}_{f}\left[\|y\|_{\beta}+\frac{\|y \otimes y\|_{2 \beta}}{\|y\|_{\beta}}\right]\right)^{1 / \beta} .
$$

The constant $k$ appearing in the definition of $\beta$ will be chosen later. Suppose that $0 \leq s<t \leq T$ satisfy the following inequalities:

$$
\begin{aligned}
(t-s)^{\beta}\|x\|_{\beta} & \leq 1, \\
(t-s)^{\beta}\|\widetilde{x}\|_{\beta} & \leq 1, \\
(t-s)^{\beta}\|x \otimes y\|_{2 \beta} & \leq\|y\|_{\beta}, \\
(t-s)^{\beta}\|\widetilde{x} \otimes \widetilde{y}\|_{2 \beta} & \leq\|\widetilde{y}\|_{\beta} .
\end{aligned}
$$

Our first purpose is to estimate the Hölder norm $\|x-\widetilde{x}\|_{s, t, \beta}$. We can write

$$
\begin{aligned}
\|x-\widetilde{x}\|_{s, t, \beta} & \leq\left\|\int\left[f\left(x_{s}\right)-f\left(\widetilde{x}_{s}\right)\right] d y_{s}\right\|_{s, t, \beta}+\left\|\int f\left(\widetilde{x}_{s}\right) d\left(y_{s}-\widetilde{y}_{s}\right)\right\|_{s, t, \beta} \\
& =I_{1, s, t}+I_{2, s, t} .
\end{aligned}
$$


The term $I_{1, s, t}$ can be estimated using (6.4) and we obtain

$$
I_{1, s, t} \leq k\left[H_{s, t}^{1}(y)\|x-\widetilde{x}\|_{s, t, \infty}+H_{s, t}^{2}(y)\|x-\widetilde{x}\|_{s, t, \beta}+H_{s, t}^{3}\|(x-\widetilde{x}) \otimes y\|_{s, t, 2 \beta}\right],
$$

where $H_{s, t}^{1}(y), H_{s, t}^{2}(y)$, and $H_{s, t}^{3}$ are defined in (6.5), (6.6), and (6.7), respectively. Then, using the inequalities (4.20), (4.21), and (4.22) we get the following estimates:

$$
\begin{aligned}
H_{s, t}^{1}(y) & \leq k\|y\|_{\beta} \widehat{\rho}_{f}, \\
H_{s, t}^{2}(y) & \leq k\|y\|_{\beta} \widehat{\rho}_{f}(t-s)^{\beta}, \\
H_{s, t}^{3} & \leq \widehat{\rho}_{f}(t-s)^{\beta} .
\end{aligned}
$$

It remains to handle the term $\|(x-\widetilde{x}) \otimes y\|_{s, t, 2 \beta}$ in (4.24). From (4.2) for the solution $(\bar{x}, \bar{y}, \bar{x} \otimes \bar{y})$ we obtain

$$
\begin{aligned}
\left|((x-\widetilde{x}) \otimes y)_{s, t}\right|= & \left|\int_{s}^{t} f\left(x_{r}\right) d_{r}(y \otimes y)_{\cdot, t}-\int_{s}^{t} f\left(\widetilde{x}_{r}\right) d_{r}(\widetilde{y} \otimes y)_{\cdot, t}\right| \\
= & \left|\int_{s}^{t}\left[f\left(x_{r}\right)-f\left(\widetilde{x}_{r}\right)\right] d_{r}(y \otimes y)_{\cdot, t}\right| \\
& +\left|\int_{s}^{t} f\left(\widetilde{x}_{r}\right) d_{r}((y-\widetilde{y}) \otimes y)_{\cdot, t}\right| \\
:= & B_{1}+B_{2} .
\end{aligned}
$$

For the term $B_{1}$ we have, using the inequality (6.4),

$$
\begin{aligned}
B_{1} \leq & k(t-s)^{\beta}\left[H_{s, t}^{1}\left((y \otimes y)_{., t}\right)\|x-\widetilde{x}\|_{s, t, \infty}+H_{s, t}^{2}\left((y \otimes y)_{., t}\right)\|x-\widetilde{x}\|_{s, t, \beta}\right. \\
& \left.+H_{s, t}^{3}\left\|(x-\widetilde{x}) \otimes(y \otimes y)_{\cdot, t}\right\|_{s, t, 2 \beta}\right] .
\end{aligned}
$$

The definition of $H_{s, t}^{1}\left((y \otimes y)_{., t}\right)$ together with the estimates (3.29) and (3.30) leads to

$$
\begin{aligned}
H_{s, t}^{1}\left((y \otimes y)_{., t}\right) \leq & \Phi_{s, t, \beta}(y, y)\left\|f^{\prime}\right\|_{\infty}(t-s)^{2 \beta} \\
& +\left(\left\|f^{\prime \prime}\right\|_{\infty}+\left\|f^{\prime \prime}\right\|_{\lambda}\left(\|x\|_{s, t, \beta}^{\lambda}+\|\widetilde{x}\|_{s, t, \beta}^{\lambda}\right)(t-s)^{\beta \lambda}\right) \\
& \times\left(\Phi_{s, t, \beta}(x, y, y)+\left(\|x\|_{s, t, \beta}+\|\widetilde{x}\|_{s, t, \beta}\right) \Phi_{s, t, \beta}(y, y)\right)(t-s)^{2 \beta},
\end{aligned}
$$

and the estimates (4.20), (4.21) and (4.22) imply

$$
H_{s, t}^{1}\left((y \otimes y)_{., t}\right) \leq k \widehat{\rho}_{f} \Phi_{\beta}(y, y)(t-s)^{\beta},
$$

where $\Phi_{\beta}(y, y)=\|y \otimes y\|_{2 \beta}+\|y\|_{\beta}^{2}$. In a similar way, the definition of $H_{s, t}^{2}\left((y \otimes y)_{,, t}\right)$ together with the estimates (3.29) and (3.30) leads to

$$
\begin{aligned}
H_{s, t}^{2}(( & \left.(y y)_{., t}\right) \leq k\left\|f^{\prime \prime}\right\|_{\infty}\left(\Phi_{s, t, \beta}(x, y, y)\right. \\
& \left.+\left(\|x\|_{s, t, \beta}+\|\widetilde{x}\|_{s, t, \beta}\right) \Phi_{s, t, \beta}(y, y)\right)(t-s)^{3 \beta} \\
& +\left\|f^{\prime}\right\|_{\infty} \Phi_{s, t, \beta}(y, y)(t-s)^{2 \beta} \\
\leq \quad & k \widehat{\rho}_{f} \Phi_{\beta}(y, y)(t-s)^{2 \beta} .
\end{aligned}
$$

On the other hand, from (3.30) we get

$$
\left\|(x-\tilde{x}) \otimes(y \otimes y)_{\cdot, t}\right\|_{s, t, 2 \beta} \leq k \Phi_{s, t}(x-\widetilde{x}, y, y)(t-s)^{\beta} .
$$


Thus, substituting (4.30), (4.31), (4.27) and (4.32) into (4.29) yields

$$
\begin{aligned}
B_{1} \leq & k \widehat{\rho}_{f}(t-s)^{2 \beta} \Phi_{\beta}(y, y)\|x-\widetilde{x}\|_{s, t, \infty} \\
& +k \widehat{\rho}_{f}(t-s)^{3 \beta} \Phi_{\beta}(y, y)\|x-\widetilde{x}\|_{s, t, \beta} \\
& +k \widehat{\rho}_{f}(t-s)^{3 \beta} \Phi_{s, t, \beta}(x-\widetilde{x}, y, y) \\
\leq & k(t-s)^{2 \beta} \widehat{\rho}_{f} \Phi_{\beta}(y, y)\left(\|x-\widetilde{x}\|_{s, t, \infty}+\|x-\widetilde{x}\|_{s, t, \beta}(t-s)^{\beta}\right) \\
& +k(t-s)^{3 \beta} \widehat{\rho}_{f}\|y\|_{\beta}\|(x-\widetilde{x}) \otimes y\|_{s, t, \beta} .
\end{aligned}
$$

For the term $B_{2}$ we obtain, using Proposition 3.9 .

$$
\begin{aligned}
B_{2} \leq & k\left|f\left(\widetilde{x}_{s}\right)\right| \Phi_{s, t, \beta}(y-\widetilde{y}, y)(t-s)^{2 \beta} \\
& +k\left(\left\|f^{\prime}\right\|_{\infty}+\left\|f^{\prime}\right\|_{\lambda}\|\widetilde{x}\|_{s, t, \beta}^{\lambda}(t-s)^{\lambda \beta}\right) \Phi_{s, t, \beta}(\widetilde{x}, y-\widetilde{y}, y)(t-s)^{3 \beta} .
\end{aligned}
$$

From (4.2) when $x$ and $y$ are replaced by $\widetilde{x}$ and $y-\widetilde{y}$ we get

$$
(\widetilde{x} \otimes(y-\widetilde{y}))_{s, t}=\int_{s}^{t} f\left(\widetilde{x}_{r}\right) d_{r}\left(\widetilde{y} \otimes(y-\widetilde{y})_{\cdot, t} .\right.
$$

As a consequence, we can estimate $\|\widetilde{x} \otimes(y-\widetilde{y})\|_{s, t, 2 \beta}$ using Proposition 3.9 and we obtain

$$
\begin{aligned}
\|\widetilde{x} \otimes(y-\widetilde{y})\|_{s, t, 2 \beta} \leq & k\left|f\left(\widetilde{x}_{s}\right)\right| \Phi_{s, t, \beta}(\widetilde{y}, y-\widetilde{y}) \\
& +k\left(\left\|f^{\prime}\right\|_{\infty}+\left\|f^{\prime}\right\|_{\lambda}\|\widetilde{x}\|_{s, t, \beta}^{\lambda}(t-s)^{\lambda \beta}\right) \\
& \times \Phi_{s, t, \beta}(\widetilde{x}, \widetilde{y}, y-\widetilde{y})(t-s)^{\beta},
\end{aligned}
$$

and using (4.21) and (4.23),

$$
\begin{aligned}
(t-s)^{\beta} \Phi_{s, t, \beta}(\widetilde{x}, \widetilde{y}, y-\widetilde{y}) & \leq\|\widetilde{y}\|_{\beta}\|y-\widetilde{y}\|_{\beta}+\|\widetilde{y} \otimes(y-\widetilde{y})\|_{2 \beta} \\
& \leq \Phi_{\beta}(\widetilde{y}, y-\widetilde{y}) .
\end{aligned}
$$

Substituting (4.36) into (4.35), and using (4.21) yields

$$
\|\widetilde{x} \otimes(y-\widetilde{y})\|_{s, t, 2 \beta} \leq k \widehat{\rho}_{f} \Phi_{\beta}(\widetilde{y}, y-\widetilde{y}) .
$$

Then, substituting (4.37) into (4.34) we obtain

$$
B_{2} \leq k \widehat{\rho}_{f}(t-s)^{2 \beta}\left[\Phi_{\beta}(y-\widetilde{y}, y)+\widehat{\rho}_{f}\|y\|_{\beta}(t-s)^{\beta} \Phi_{\beta}(\widetilde{y}, y-\widetilde{y})\right] .
$$

From (4.33), (4.38) and (4.28) we get

$$
\begin{aligned}
\|(x-\widetilde{x}) \otimes y\|_{s, t, 2 \beta} \leq & k \widehat{\rho}_{f} \Phi_{\beta}(y, y)\left(\|x-\widetilde{x}\|_{s, t, \infty}+\|x-\widetilde{x}\|_{s, t, \beta}(t-s)^{\beta}\right) \\
& +k \widehat{\rho}_{f}\|y\|_{\beta}\|(x-\widetilde{x}) \otimes y\|_{s, t, 2 \beta}(t-s)^{\beta} \\
& +k \widehat{\rho}_{f} \Phi_{\beta}(y-\widetilde{y}, y) \\
& +k\left(\widehat{\rho}_{f}\right)^{2}\|y\|_{\beta} \Phi_{\beta}(\widetilde{y}, y-\widetilde{y})(t-s)^{\beta} .
\end{aligned}
$$

The condition $t-s \leq 1 / \beta(y)$, if the constant $k$ in $\beta(y)$ is chosen in an appropriate way, implies that

Hence, assuming $k \geq 2$, we have

$$
k(t-s)^{\beta} \widehat{\rho}_{f}\|y\|_{\beta} \leq \frac{1}{2} .
$$

$$
\begin{aligned}
\|(x-\widetilde{x}) \otimes y\|_{s, t, 2 \beta} \leq & k \widehat{\rho}_{f} \Phi_{\beta}(y, y)\left(\|x-\widetilde{x}\|_{s, t, \infty}+\|x-\widetilde{x}\|_{s, t, \beta}(t-s)^{\beta}\right) \\
& +k \widehat{\rho}_{f}\left[\Phi_{\beta}(y-\widetilde{y}, y)+\widehat{\rho}_{f} \Phi_{\beta}(\widetilde{y}, y-\widetilde{y})\right] .
\end{aligned}
$$


Substituting (4.25), (4.26), (4.27) and (4.39) into (4.24) yields

$$
\begin{aligned}
I_{1, s, t} \leq & k \widehat{\rho}_{f}\|y\|_{\beta}\left(\|x-\widetilde{x}\|_{s, t, \infty}+\|x-\widetilde{x}\|_{s, t, \beta}(t-s)^{\beta}\right) \\
& +k\left(\widehat{\rho}_{f}\right)^{2} \Phi_{\beta}(y, y)\left(\|x-\widetilde{x}\|_{s, t, \infty}+\|x-\widetilde{x}\|_{s, t, \beta}(t-s)^{\beta}\right)(t-s)^{\beta} \\
& +k\left(\widehat{\rho}_{f}\right)^{2}\left(\Phi_{\beta}(y-\widetilde{y}, y)+\widehat{\rho}_{f} \Phi_{\beta}(\widetilde{y}, y-\widetilde{y})\right)(t-s)^{\beta} .
\end{aligned}
$$

Again, condition $t-s \leq 1 / \beta(y)$, if the constant $k$ in $\beta(y)$ is chosen in an appropriate way, implies that

$$
\begin{aligned}
I_{1, s, t} \leq & k \widehat{\rho}_{f}\|y\|_{\beta}\|x-\widetilde{x}\|_{s, t, \infty}+\frac{1}{2}\|x-\widetilde{x}\|_{s, t, \beta} \\
& +k\left(\widehat{\rho}_{f}\right)^{2}\left(\Phi_{\beta}(y-\widetilde{y}, y)+\widehat{\rho}_{f} \Phi_{\beta}(\widetilde{y}, y-\widetilde{y})\right)(t-s)^{\beta} .
\end{aligned}
$$

For the term $I_{2, s, t}$ we have the following estimates, using (3.16), (4.21) and (4.37):

$$
\begin{aligned}
I_{2, s, t \leq} \leq & k\|f\|_{\infty}\|y-\widetilde{y}\|_{s, t, \beta}+k \Phi_{s, t, \beta}(\widetilde{x}, y-\widetilde{y}) \\
& \times\left(\left\|f^{\prime}\right\|_{\infty}+\left\|f^{\prime}\right\|_{\lambda}\|\widetilde{x}\|_{s, t, \beta}^{\lambda}(t-s)^{\lambda \beta}\right)(t-s)^{\beta} \\
\leq & k \widehat{\rho}_{f}\left(\|y-\widetilde{y}\|_{\beta}+\|\widetilde{x} \otimes(y-\tilde{y})\|_{s, t, 2 \beta}(t-s)^{\beta}\right) \\
\leq & k \widehat{\rho}_{f}\left(\|y-\widetilde{y}\|_{\beta}+\widehat{\rho}_{f} \Phi_{\beta}(\widetilde{y}, y-\widetilde{y})(t-s)^{\beta}\right) .
\end{aligned}
$$

Now from (4.40) and (4.41) we get

$$
\|x-\widetilde{x}\|_{s, t, \beta} \leq k \widehat{\rho}_{f}\|y\|_{\beta}\|x-\widetilde{x}\|_{s, t, \infty}+\frac{1}{2}\|x-\widetilde{x}\|_{s, t, \beta}+\Psi(y, \widetilde{y}),
$$

where

$$
\begin{aligned}
\Psi(y, \widetilde{y}) & =k\left(\widehat{\rho}_{f}\right)^{2}\left(\Phi_{\beta}(y-\widetilde{y}, y)+\widehat{\rho}_{f} \Phi_{\beta}(\widetilde{y}, y-\widetilde{y})\right)(t-s)^{\beta}+k \widehat{\rho}_{f}\|y-\widetilde{y}\|_{\beta} \\
& \leq K\left(\|y-\widetilde{y}\|_{\beta}+\|(y-\widetilde{y}) \otimes y\|_{2 \beta}+\|\widetilde{y} \otimes(y-\widetilde{y})\|_{2 \beta}\right),
\end{aligned}
$$

for some constant $K$, depending on $\|y\|_{\beta},\|\widetilde{y}\|_{\beta},\|y \otimes y\|_{2 \beta}, \beta, \lambda$, and $\widehat{\rho}_{f}$. Hence,

$$
\|x-\widetilde{x}\|_{s, t, \beta} \leq k \widehat{\rho}_{f}\|y\|_{\beta}\|x-\widetilde{x}\|_{s, t, \infty}+2 \Psi(y, \widetilde{y}) .
$$

Notice that

$$
\|x-\widetilde{x}\|_{s, t, \infty} \leq\left|x_{s}-\widetilde{x}_{s}\right|+(t-s)^{\beta}\|x-\widetilde{x}\|_{s, t, \beta}
$$

Hence,

$$
\|x-\widetilde{x}\|_{s, t, \beta} \leq k \widehat{\rho}_{f}\|y\|_{\beta}\left[\left|x_{s}-\widetilde{x}_{s}\right|+(t-s)^{\beta}\|x-\widetilde{x}\|_{s, t, \beta}\right]+2 \Psi(y, \widetilde{y}) .
$$

Consequently,

$$
\|x-\widetilde{x}\|_{s, t, \beta} \leq k \widehat{\rho}_{f}\|y\|_{\beta}\left|x_{s}-\widetilde{x}_{s}\right|+4 \Psi(y, \widetilde{y}) .
$$

Substituting (4.44) into (4.43) yields

$$
\|x-\widetilde{x}\|_{s, t, \infty} \leq\left|x_{s}-\widetilde{x}_{s}\right|+(t-s)^{\beta} k \widehat{\rho}_{f}\|y\|_{\beta}\left|x_{s}-\tilde{x}_{s}\right|+4(t-s)^{\beta} \Psi(y, \widetilde{y}) .
$$

Denote $\delta=\frac{1}{\beta(y)}$ and $t_{n}=n \delta$. Set

$$
Z_{n}=\sup _{0 \leq s \leq t_{n}}\left|x_{s}-\widetilde{x}_{s}\right| .
$$

Then inequality (4.45) states that

$$
Z_{n+1} \leq\left(1+k \widehat{\rho}_{f} \delta^{\beta}\right) Z_{n}+4 \delta^{\beta} \Psi(y, \widetilde{y}) .
$$


Therefore

$$
\begin{aligned}
Z_{T} \leq & \left(1+k \widehat{\rho}_{f} \delta^{\beta}\right)^{\left[\frac{T}{\delta}\right]}\left|x_{0}-\widetilde{x}_{0}\right| \\
+ & +k \sum_{l=0}^{\left[\frac{T}{\delta}\right]}\left(1+k \widehat{\rho}_{f} \delta^{\beta}\right)^{l} 4 \delta^{\beta} \Psi(y, \widetilde{y}) .
\end{aligned}
$$

This implies the desired estimate.

The following corollary is a direct consequence of (4.42) and (4.18).

Corollary 4.3. Under the assumptions of Theorem 4.2, if $x$ and $\tilde{x}$ satisfy

$$
x_{t}=x_{0}+\int_{0}^{t} f\left(x_{s}\right) d y_{s} \quad \text { and } \quad \tilde{x}_{t}=\tilde{x}_{0}+\int_{0}^{t} f\left(\tilde{x}_{s}\right) d \tilde{y}_{s}
$$

then

$$
\left.\left\|x_{t}-\tilde{x}_{t}\right\|_{\beta} \leq C\left\{\left|x_{0}-\tilde{x}_{0}\right|+\|y-\tilde{y}\|_{\beta}+[\|(y-\tilde{y}) \otimes y)\left\|_{2 \beta}+\right\| \tilde{y} \otimes(y-\tilde{y}) \|_{2 \beta}\right]\right\} .
$$

\section{Stochastic Differential Equations}

Suppose that $B=\left\{B_{t}=\left(B_{t}^{1}, B_{t}^{2}, \ldots, B_{t}^{d}\right), t \geq 0\right\}$ is a $d$-dimensional Brownian motion. Fix a time interval $[0, T]$. Define

$$
(B \otimes B)_{s, t}=\int_{s}^{t}\left(B_{r}-B_{s}\right) d^{\circ} B_{r}
$$

where the stochastic integral is a Stratonovich integral. That is,

$$
(B \otimes B)_{s, t}^{i, j}= \begin{cases}\frac{1}{2}\left(B_{t}^{i}-B_{s}^{i}\right)^{2} & \text { if } i=j, \\ \int_{s}^{t}\left(B_{r}^{i}-B_{s}^{i}\right) d B_{r}^{j} & \text { if } i \neq j,\end{cases}
$$

where the stochastic integral is an Itô integral. It is not difficult to show that we can choose a version of $(B \otimes B)_{s, t}$ in such a way that $(B, B, B \otimes B)$ constitutes a $\beta$-Hölder continuous multiplicative functional, for a fixed $\beta \in(1 / 3,1 / 2)$.

As a first application of Theorem 3.3 and (3.11) we deduce that the Stratonovich stochastic integral $\int_{0}^{T} f\left(B_{r}\right) d^{\circ} B_{r}$ has the following path-wise expression:

$$
\begin{aligned}
\int_{0}^{T} f\left(B_{r}\right) d^{\circ} B_{r}= & (-1)^{\alpha} \sum_{j=1}^{d} \int_{0}^{T} \widehat{D}_{0+}^{\alpha} f_{j}(B)_{r}\left(D_{T-}^{1-\alpha} B_{T-}^{j}\right)_{r} d r \\
& -(-1)^{2 \alpha-1} \sum_{i, j=1}^{d} \int_{0}^{T} D_{0+}^{2 \alpha-1} \partial_{i} f_{j}(B)_{r} D_{T-}^{1-\alpha} \mathcal{D}_{T-}^{1-\alpha}(B \otimes B)^{i, j} d r .
\end{aligned}
$$

In the same way, if we set $(B \widehat{\otimes} B)_{s, t}^{i, j}=(B \otimes B)_{s, t}^{i, j}-\frac{1}{2}(t-s) \delta_{i, j}$, then the path-wise integral $\int_{0}^{T} f\left(B_{r}\right) \widehat{d} B_{r}$ corresponding to the multiplicative functional $(B, B \widehat{\otimes} B)$ is the Itô integral:

$$
\begin{aligned}
\int_{0}^{T} f\left(B_{r}\right) \widehat{d} B_{r}= & (-1)^{\alpha} \sum_{j=1}^{d} \int_{0}^{T} \widehat{D}_{0+}^{\alpha} f_{j}(B)_{r}\left(D_{T-}^{1-\alpha} B_{T-}^{j}\right)_{r} d r \\
& -(-1)^{2 \alpha-1} \sum_{i, j=1}^{d} \int_{0}^{T} D_{0+}^{2 \alpha-1} \partial_{i} f_{j}(B)_{r} D_{T-}^{1-\alpha} \mathcal{D}_{T-}^{1-\alpha}(B \widehat{\otimes} B)^{i, j} d r
\end{aligned}
$$


Notice that Proposition 3.5 yields the well-known relation between both integrals:

$$
\int_{0}^{T} f\left(B_{r}\right) d^{\circ} B_{r}-\int_{0}^{T} f\left(B_{r}\right) \widehat{d} B_{r}=\frac{1}{2} \sum_{i=1}^{d} \int_{0}^{T} \partial_{i} f_{i}\left(B_{r}\right) d r .
$$

We can apply Theorem 4.1 and deduce the existence of a solution for the stochastic differential equation in $\mathbb{R}^{m}$ :

$$
X_{t}=X_{0}+\int_{0}^{t} f\left(X_{s}\right) d^{\circ} B_{s},
$$

where the initial condition $X_{0}$ is an arbitrary random variable, and the function $f: \mathbb{R}^{m} \rightarrow \mathbb{R}^{m d}$ is a continuously differentiable function such that $f^{\prime}$ is $\lambda$-Hölder continuous, where $\lambda>\frac{1}{\beta}-2$, and $f$ and $f^{\prime}$ are bounded. By Theorem 4.2 the solution is unique if $f$ is twice continuously differentiable with bounded derivatives and $f^{\prime \prime}$ is $\lambda$-Hölder continuous, where $\lambda>\frac{1}{\beta}-2$. The stochastic integral here is a path-wise integral which depends on $B$ and $B \otimes B$.

We have also the stability-type results (4.18) and (4.46). In particular, assume that $B^{\varepsilon}$ is a piece-wise smooth approximation of $B$ such that

$$
\left\|B-B^{\varepsilon}\right\|_{\beta},\left\|B^{\varepsilon} \otimes\left(B-B^{\varepsilon}\right)\right\|_{2 \beta}, \text { and }\left\|\left(B-B^{\varepsilon}\right) \otimes B\right\|_{2 \beta}
$$

converge to zero with a certain rate. Let $\bar{X}=\left(X, X^{\varepsilon}\right)$ be the solution of

$$
\bar{X}_{t}=\bar{X}_{0}+\int_{0}^{t} \bar{f}\left(\bar{X}_{s}\right) d \bar{B}_{s},
$$

where $\bar{X}_{0}=\left(X_{0}, X_{0}\right), \bar{B}=\left(B, B^{\varepsilon}\right)$, and we use the notation of Theorem 4.2. Then according to Corollary 4.3 . $\left\|X-X^{\varepsilon}\right\|_{\beta}$ will also converge to 0 with the same rate. In particular, this implies that the stochastic process $X$ coincides with the solution of the Stratonovich stochastic differential equation

$$
X_{t}=X_{0}+\int_{0}^{t} f\left(X_{s}\right) d^{\circ} B_{s}^{H} .
$$

In this section we will apply these results in order to obtain the almost sure rate of convergence of the Wong-Zakai approximation to the stochastic differential equation (5.1). That is, we will consider the rate of convergence in the Hölder norm when we approximate the Brownian motion by a polygonal line.

In order to get a precise rate for these approximations we will make use of the following exact modulus of continuity of the Brownian motion. There exists a random variable $G$ such that almost surely for any $s, t \in[0, T]$ we have

$$
\left|B_{t}-B_{s}\right| \leq G|t-s|^{1 / 2} \sqrt{\log \left(|t-s|^{-1}\right)} .
$$

Let $\pi=\left\{0=t_{0}<t_{1}<\cdots<t_{n}=T\right\}$ be the uniform partition of the interval $[0, T]$. That is, $t_{k}=\frac{k T}{n}, k=0, \ldots, n$. We denote by $B^{\pi}$ the polygonal approximation of the Brownian motion defined by

$$
B_{t}^{\pi}=\sum_{k=0}^{n-1}\left(B_{t_{k}}+\frac{n}{T}\left(t-t_{k}\right)\left(B_{t_{k+1}}-B_{t_{k}}\right)\right) \mathbf{1}_{\left(t_{k}, t_{k+1}\right]}(t) .
$$

We have the following result. 
Lemma 5.1. There exists a random variable $C_{T, \beta}$ such that

$$
\begin{aligned}
\left\|B-B^{\pi}\right\|_{\beta} & \leq C_{T, \beta} n^{\beta-1 / 2} \sqrt{\log n} \\
\left\|B \otimes\left(B-B^{\pi}\right)\right\|_{2 \beta} & \leq C_{T, \beta} n^{\beta-1 / 2} \sqrt{\log n} \\
\left\|B^{\pi} \otimes\left(B-B^{\pi}\right)\right\|_{2 \beta} & \leq C_{T, \beta} n^{\beta-1 / 2} \sqrt{\log n} \\
\left\|\left(B-B^{\pi}\right) \otimes B\right\|_{2 \beta} & \leq C_{T, \beta} n^{\beta-1 / 2} \sqrt{\log n} .
\end{aligned}
$$

Proof. Fix $0<s<t<T$ and assume that $s \in\left[t_{l}, t_{l+1}\right]$ and $t \in\left[t_{k}, t_{k+1}\right]$. Let us first estimate

$$
h_{1}(s, t)=\frac{1}{(t-s)^{\beta}}\left|B_{t}^{\pi}-B_{t}-\left(B_{s}^{\pi}-B_{s}\right)\right| .
$$

If $t-s \geq \frac{T}{n}$, then using (5.3) we obtain

$$
\begin{aligned}
\left|h_{1}(s, t)\right| \leq & T^{-\beta} n^{\beta}\left[\left|B_{t_{k}}-B_{t}+\frac{n}{T}\left(t-t_{k}\right)\left(B_{t_{k+1}}-B_{t_{k}}\right)\right|\right. \\
& \left.+\left|B_{t_{l}}-B_{s}+\frac{n}{T}\left(s-t_{l}\right)\left(B_{t_{l+1}}-B_{t_{l}}\right)\right|\right] \\
\leq & 4 G T^{-\beta+1 / 2} n^{-1 / 2+\beta} \sqrt{\log (n / T)} .
\end{aligned}
$$

If $t-s<\frac{T}{n}$, then there are two cases. Suppose first that $s, t \in\left[t_{k}, t_{k+1}\right]$. In this case, if $n$ is large enough $\left(n>T e^{2 /(1-2 \beta)}\right)$ we obtain using (5.3),

$$
\begin{aligned}
\left|h_{1}(s, t)\right| & \leq \frac{\left|B_{t}-B_{s}\right|}{(t-s)^{\beta}}+\frac{n}{T} \frac{\left|B_{t_{k+1}}-B_{t_{k}}\right|}{(t-s)^{\beta}}(t-s) \\
& \leq G|t-s|^{\frac{1}{2}-\beta} \sqrt{\log |t-s|^{-1}}+G T^{-1 / 2} \sqrt{\log (n / T)} n^{1-1 / 2}(t-s)^{1-\beta} \\
& \leq G T^{-\beta+1 / 2} n^{-1 / 2+\beta} \sqrt{\log (n / T)} .
\end{aligned}
$$

On the other hand, if $s \in\left[t_{k-1}, t_{k}\right]$ and $t \in\left[t_{k}, t_{k+1}\right]$ we have, again if $n$ is large enough,

$$
\begin{aligned}
\left|h_{1}(s, t)\right| \leq & \frac{1}{(t-s)^{\beta}} \mid B_{t_{k}}-B_{t}+\frac{n}{T}\left(t-t_{k}\right)\left(B_{t_{k+1}}-B_{t_{k}}\right) \\
& -\left\{B_{t_{k}}-B_{s}-\frac{n}{T}\left(t_{k}-s\right)\left(B_{t_{k}}-B_{t_{k-1}}\right)\right\} \mid \\
\leq & \frac{1}{(t-s)^{\beta}}\left[\left|B_{t}-B_{s}\right|+\frac{n}{T}(t-s)\left(\left|B_{t_{k}}-B_{t_{k-1}}\right|+\left|B_{t_{k+1}}-B_{t_{k}}\right|\right)\right] \\
\leq & \frac{G}{(t-s)^{\beta}}\left[|t-s|^{1 / 2} \sqrt{\log |t-s|^{-1}}+2(t-s)\left(\frac{n}{T}\right)^{1 / 2} \sqrt{\log (n / T)}\right] \\
\leq & 3 G T^{-\beta+1 / 2} n^{-1 / 2+\beta} \sqrt{\log (n / T)} .
\end{aligned}
$$

This proves (5.4).

Now we turn to the estimate of the term

$$
h_{2}(s, t)=\frac{1}{(t-s)^{2 \beta}}\left|\int_{s}^{t}\left(B_{u}^{i}-B_{s}^{i}\right) d B_{u}^{j, \pi}-\int_{s}^{t}\left(B_{u}^{i}-B_{s}^{i}\right) d B_{u}^{j}\right|
$$

for $i \neq j$ (the case $i=j$ is obvious from (5.4)). We claim that there exists a random variable $Z$ such that, almost surely, for all $s, t \in[0, T]$ we have

$$
\left|\int_{s}^{t}\left(B_{u}^{i}-B_{s}^{i}\right) d B_{u}^{j}\right| \leq Z|t-s| \log |t-s|^{-1} \text {. }
$$


In fact, it suffices to show this inequality almost surely for all $s$ and $t$ rational numbers. If we fix $s$, the process $\left\{M_{t}, t \in[s, T]\right\}$,

$$
M_{t}=\int_{s}^{t}\left(B_{u}^{i}-B_{s}^{i}\right) d B_{u}^{j}
$$

is a continuous martingale and it can be represented as a time-changed Brownian motion:

$$
M_{t}=W_{\int_{s}^{t}\left(B_{u}^{i}-B_{s}^{i}\right)^{2} d u}
$$

As a consequence, applying (5.3) there exists a random variable $G_{1}$ such that

$$
\left|M_{t}\right|=\left|W_{\int_{s}^{t}\left(B_{u}^{i}-B_{s}^{i}\right)^{2} d u}\right| \leq G\left(\int_{s}^{t}\left(B_{u}^{i}-B_{s}^{i}\right)^{2} d u\right)^{1 / 2} \sqrt{\log \left(\int_{s}^{t}\left(B_{u}^{i}-B_{s}^{i}\right)^{2} d u\right)^{-1}}
$$

and again (5.3), applied to $B_{u}^{i}-B_{s}^{i}$, yields

$$
\left|M_{t}\right| \leq G_{1} G_{2}\left(\int_{s}^{t}(u-s) \log |u-s|^{-1} d u\right)^{1 / 2} \sqrt{\log \left(G_{2}^{2} \int_{s}^{t}(u-s) \log |u-s|^{-1} d u\right)^{-1}}
$$

for some random variable $G_{2}$. We have for $|t-s| \leq 1$,

$$
\int_{s}^{t}(u-s) \log |u-s|^{-1} d u=|t-s|^{2}\left(\frac{1}{4}+\frac{1}{2} \log |t-s|^{-1}\right),
$$

and this implies easily the estimate (5.8).

Suppose first that $t-s \geq \frac{T}{n}$. Then

$$
\begin{aligned}
& h_{2}(t, s)= \frac{1}{(t-s)^{2 \beta}}\left|\int_{s}^{t}\left(B_{u}^{i}-B_{s}^{i}\right) d B_{u}^{j, \pi}-\int_{s}^{t}\left(B_{u}^{i}-B_{s}^{i}\right) d B_{u}^{j}\right| \\
&= \frac{1}{(t-s)^{2 \beta}} \mid\left(B_{t}^{i}-B_{s}^{i}\right)\left(B_{t}^{j, \pi}-B_{s}^{j, \pi}\right)-\int_{s}^{t}\left(B_{u}^{j, \pi}-B_{s}^{j, \pi}\right) d B_{u}^{i} \\
& \quad-\left(B_{t}^{i}-B_{s}^{i}\right)\left(B_{t}^{j}-B_{s}^{j}\right)+\int_{s}^{t}\left(B_{u}^{j}-B_{s}^{j}\right) d B_{u}^{i} \mid \\
& \leq \frac{1}{(t-s)^{2 \beta}}\left|\left(B_{t}^{i}-B_{s}^{i}\right)\left(B_{t}^{j, \pi}-B_{s}^{j, \pi}-B_{t}^{j}-B_{s}^{j}\right)\right| \\
&+\frac{1}{(t-s)^{2 \beta}}\left|\int_{s}^{t}\left[B_{u}^{j, \pi}-B_{u}^{j}\right] d B_{u}^{i}\right| \\
&= A_{1}+A_{2} .
\end{aligned}
$$

Using (5.3) and (5.4) the term $A_{1}$ can be estimated as follows:

$$
\begin{aligned}
A_{1} & \leq G|t-s|^{1 / 2-\beta} \sqrt{\log |t-s|^{-1}}\left\|B^{j, \pi}-B^{j}\right\|_{\beta} \\
& \leq C_{T, \beta} n^{\beta-1 / 2} \sqrt{\log n} .
\end{aligned}
$$

For the term $A_{2}$ we proceed as in the proof of the estimate (5.8). We have

$$
\int_{s}^{t}\left[B_{u}^{j, \pi}-B_{u}^{j}\right] d B_{u}^{i}=W_{\int_{s}^{t}\left(B_{u}^{j, \pi}-B_{u}^{j}\right)^{2} d u},
$$

where $W$ is a Brownian motion. As a consequence, using that

$$
\left\|B-B^{\pi}\right\|_{\infty} \leq C_{T, \beta} n^{-1 / 2} \sqrt{\log (n / T)}
$$


(this estimate is proved as (5.4)) we get

$$
\begin{aligned}
A_{2} & \leq \frac{G}{(t-s)^{2 \beta}}\left(\int_{s}^{t}\left(B_{u}^{j, \pi}-B_{u}^{j}\right)^{2} d u\right)^{1 / 2} \sqrt{\log \left(\int_{s}^{t}\left(B_{u}^{j, \pi}-B_{u}^{j}\right)^{2} d u\right)^{-1}} \\
& \leq C_{T, \beta}(t-s)^{1 / 2-2 \beta} n^{-1 / 2} \sqrt{\log n} \sqrt{\log \left[(t-s)^{-1} n(\log n)^{-1}\right]} \\
& \leq C_{T, \beta} n^{\beta-1 / 2} \sqrt{\log n} .
\end{aligned}
$$

Suppose now that $t-s<\frac{T}{n}$. We make the decomposition

$$
\begin{aligned}
h_{2}(s, t) & \leq \frac{1}{(t-s)^{2 \beta}}\left(\left|\int_{s}^{t}\left(B_{u}^{i}-B_{s}^{i}\right) d B_{u}^{j, \pi}\right|+\left|\int_{s}^{t}\left(B_{u}^{i}-B_{s}^{i}\right) d B_{u}^{j}\right|\right) \\
& =B_{1}+B_{2} .
\end{aligned}
$$

Then (5.8) yields

$$
B_{2} \leq Z|t-s|^{1-2 \beta} \log |t-s|^{-1} \leq C_{T, \beta} n^{2 \beta-1} \log n .
$$

In order to handle the term $B_{1}$, assume first that $s, t \in\left[t_{k}, t_{k+1}\right]$. Then

$$
\int_{s}^{t}\left(B_{u}^{i}-B_{s}^{i}\right) d B_{u}^{j, \pi}=\frac{n}{T}\left(B_{t_{k+1}}^{j}-B_{t_{k}}^{j}\right) \int_{s}^{t}\left(B_{u}^{i}-B_{s}^{i}\right) d u
$$

and we obtain

$$
B_{1} \leq C_{T, \beta}(t-s)^{1-2 \beta} \log n \leq C_{T, \beta} n^{1-2 \beta} \log n .
$$

Finally, if $s \in\left[t_{k-1}, t_{k}\right]$ and $t \in\left[t_{k}, t_{k+1}\right]$ we have

$$
\begin{aligned}
B_{1} \leq & (t-s)^{-2 \beta} \frac{n}{T} \mid\left(B_{t_{k}}^{j}-B_{t_{k-1}}^{j}\right) \int_{s}^{t_{k}}\left(B_{u}^{i}-B_{s}^{i}\right) d u \\
& \quad+\left(B_{t_{k+1}}^{j}-B_{t_{k}}^{j}\right) \int_{t_{k}}^{t}\left(B_{u}^{i}-B_{s}^{i}\right) d u \mid \\
\leq &
\end{aligned}
$$

The inequality (5.6) can be proved similarly, and (5.7) is a direct consequence of (5.10). The proof is now complete.

As a consequence, we can establish the following result.

Theorem 5.2. Let $f: \mathbb{R}^{m} \rightarrow \mathbb{R}^{m d}$ be continuously differentiable with bounded derivative up to fourth order and let $X$ satisfy

$$
X_{t}=X_{0}+\int_{0}^{t} f\left(X_{s}\right) d^{\circ} B_{s}
$$

If $X_{t}^{\pi}$ satisfies the following ordinary differential equation,

$$
X_{t}^{\pi}=X_{0}+\int_{0}^{t} f\left(X_{s}^{\pi}\right) d B_{s}^{\pi}
$$

then for any $\beta \in(1 / 3,1 / 2)$, there is a random constant $C_{T, \beta} \in(0, \infty)$ such that

$$
\left\|X-X^{\pi}\right\|_{\beta} \leq C_{T, \beta} n^{\beta-1 / 2} \sqrt{\log n} .
$$

Proof. The result is a straightforward consequence of Lemma 5.1 and Theorem 4.2 . 


\section{ApPEndix}

Lemma 6.1. We have for any $x<y$,

$$
x^{1-\alpha}-y^{1-\alpha} \leq(y-x)^{\beta} x^{1-\alpha-\beta} .
$$

Proof. Suppose first that $y-x \leq x$. Then

$$
x^{1-\alpha}-y^{1-\alpha}=(1-\alpha) \int_{x}^{y} u^{-\alpha} d u \leq(y-x) x^{-\alpha} \leq(y-x)^{\beta} x^{1-\alpha-\beta} .
$$

On the other hand, if $y-x \geq x$ we can write

$$
x^{1-\alpha}-y^{1-\alpha} \leq x^{\beta}\left[x^{1-\alpha-\beta}-y^{1-\alpha-\beta}\right] \leq(y-x)^{\beta} x^{1-\alpha-\beta} .
$$

Lemma 6.2. For any $z \in[0,1]$ we have

$$
\int_{0}^{1} x^{2 \beta}\left(x^{\alpha-2}-(x+z)^{\alpha-2}\right) d x \leq \frac{2-2^{\alpha-2}}{2 \beta+\alpha-1} z^{2 \beta+\alpha-1} .
$$

Proof. We can decompose the integral as follows:

$$
\begin{aligned}
\int_{0}^{1} x^{2 \beta}\left(x^{\alpha-2}-(x+z)^{\alpha-2}\right) d x= & \int_{0}^{z} x^{2 \beta}\left(x^{\alpha-2}-(x+z)^{\alpha-2}\right) d x \\
& +\int_{z}^{1} x^{2 \beta}\left(x^{\alpha-2}-(x+z)^{\alpha-2}\right) d x \\
\leq & \int_{0}^{z} x^{2 \beta+\alpha-2} d x+\int_{z}^{1} x^{2 \beta}\left(x^{\alpha-2}-(2 x)^{\alpha-2}\right) d x \\
= & \frac{2-2^{\alpha-2}}{2 \beta+\alpha-1} z^{2 \beta+\alpha-1} .
\end{aligned}
$$

Lemma 6.3. For any $a \leq b \leq T$ we have

$$
\left\|\mathcal{D}_{b-}^{1-\alpha}(x \otimes y)\right\|_{a, b, \beta} \leq k \Phi_{a, b, \beta}(x, y)(b-a)^{\beta+\alpha-1},
$$

where the constant $k$ depends on $\alpha$ and $\beta$.

Proof. Let us first consider the first term in the definition of $\mathcal{D}_{b-}^{1-\alpha}(x \otimes y)$. Fix $a \leq s<t \leq b$. We have

$$
\begin{aligned}
\left|\frac{(x \otimes y)_{t, b}}{(b-t)^{1-\alpha}}-\frac{(x \otimes y)_{s, b}}{(b-s)^{1-\alpha}}\right|= & \left|\frac{(x \otimes y)_{t, b}(b-s)^{1-\alpha}-(x \otimes y)_{s, b}(b-t)^{1-\alpha}}{(b-t)^{1-\alpha}(b-s)^{1-\alpha}}\right| \\
\leq & \frac{\left|(x \otimes y)_{t, b}\right|\left[(b-t)^{1-\alpha}-(b-s)^{1-\alpha}\right]}{(b-t)^{1-\alpha}(b-s)^{1-\alpha}} \\
& +\frac{\left|(x \otimes y)_{t, b}-(x \otimes y)_{s, b}\right|}{(b-s)^{1-\alpha}} \\
= & A_{1}+A_{2} .
\end{aligned}
$$

Using (3.2) yields

$$
A_{1} \leq\|x \otimes y\|_{t, b, 2 \beta}(b-t)^{2 \beta+a-1}(b-s)^{\alpha-1}\left[(b-t)^{1-\alpha}-(b-s)^{1-\alpha}\right],
$$


and (3.1) together with (3.2) implies

$$
\begin{aligned}
A_{2} & \leq\left(\left|(x \otimes y)_{s, t}\right|+\left|\left(x_{t}-x_{s}\right)\left(y_{b}-y_{t}\right)\right|\right)(b-s)^{\alpha-1} \\
& \leq\|x \otimes y\|_{s, t, 2 \beta}(t-s)^{2 \beta}(b-s)^{\alpha-1}+\|x\|_{s, t, \beta}\|y\|_{t, b, \beta}(t-s)^{\beta}(b-s)^{\alpha-1}(b-t)^{\beta} \\
& \leq \Phi_{a, b, \beta}(x, y)(t-s)^{\beta}(b-s)^{\alpha+\beta-1} .
\end{aligned}
$$

Then, (6.1) implies

$$
A_{1} \leq\|x \otimes y\|_{a, b, 2 \beta}(b-s)^{\alpha+\beta-1}(t-s)^{\beta} .
$$

Consider now the second term in the definition of $\mathcal{D}_{b-}^{1-\alpha}(x \otimes y)$. Fix $a \leq s<t \leq b$. We have

$$
\begin{aligned}
& \left|\int_{t}^{b} \frac{(x \otimes y)_{t, \theta}}{(\theta-t)^{2-\alpha}} d \theta-\int_{s}^{b} \frac{(x \otimes y)_{s, \theta}}{(\theta-s)^{2-\alpha}} d \theta\right| \\
\leq & \int_{s}^{t} \frac{\left|(x \otimes y)_{s, \theta}\right|}{(\theta-s)^{2-\alpha}} d \theta+\int_{t}^{b}\left|\frac{(x \otimes y)_{s, \theta}}{(\theta-s)^{2-\alpha}}-\frac{(x \otimes y)_{t, \theta}}{(\theta-t)^{2-\alpha}}\right| d \theta \\
= & B_{1}+B_{2} .
\end{aligned}
$$

For the term $B_{1}$ we have using (3.2),

$$
B_{1} \leq \frac{\|x \otimes y\|_{s, t, 2 \beta}}{2 \beta+\alpha-1}(t-s)^{2 \beta+\alpha-1} .
$$

For the term $B_{2}$ we write

$$
\begin{aligned}
B_{2}= & \int_{t}^{b}\left|\frac{(x \otimes y)_{s, \theta}(\theta-t)^{2-\alpha}-(x \otimes y)_{t, \theta}(\theta-s)^{2-\alpha}}{(\theta-s)^{2-\alpha}(\theta-t)^{2-\alpha}}\right| d \theta \\
\leq & \int_{t}^{b}\left|(x \otimes y)_{t, \theta}\right|\left[(\theta-t)^{\alpha-2}-(\theta-s)^{\alpha-2}\right] d \theta \\
& +\int_{t}^{b} \frac{\left|(x \otimes y)_{t, \theta}-(x \otimes y)_{s, \theta}\right|}{(\theta-s)^{2-\alpha}} d \theta \\
= & B_{21}+B_{22} .
\end{aligned}
$$

The term $B_{21}$ can be estimated using (3.2):

$$
B_{21} \leq\|x \otimes y\|_{t, b, 2 \beta} \int_{t}^{b}(\theta-t)^{2 \beta}\left[(\theta-t)^{\alpha-2}-(\theta-s)^{\alpha-2}\right] d \theta .
$$

Making the change of variables $\frac{\theta-t}{b-t}=x$ and $\frac{s-t}{b-t}=z$, and using the estimate (6.2) yields

$$
\begin{aligned}
& \int_{t}^{b}(\theta-t)^{2 \beta}\left[(\theta-t)^{\alpha-2}-(\theta-s)^{\alpha-2}\right] d \theta \\
= & (b-t)^{2 \beta+\alpha-1} \int_{0}^{1} x^{2 \beta}\left(x^{\alpha-2}-(x+z)^{\alpha-2}\right) d x \\
\leq & \frac{2-2^{\alpha-2}}{2 \beta+\alpha-1}(t-s)^{2 \beta+\alpha-1} .
\end{aligned}
$$


Finally, using (3.1) and (3.2) we obtain

$$
\begin{aligned}
B_{22} & \leq \int_{t}^{b} \frac{\left|(x \otimes y)_{s, t}\right|+\left|x_{t}-x_{s}\right|\left|y_{\theta}-y_{t}\right|}{(\theta-s)^{2-\alpha}} d \theta \\
& \leq\left(\frac{\|x \otimes y\|_{s, b, 2 \beta}}{1-\alpha}+\frac{\|x\|_{s, t, \beta}\|y\|_{t, b, \beta}}{\alpha+\beta-1}\right)(t-s)^{2 \beta+\alpha-1} .
\end{aligned}
$$

The proof of the result is now complete.

The next two propositions provide stability results for the integral $\int f(x) d y$ on the variables $f$ and $x$.

Proposition 6.4. Suppose that $(x, y, x \otimes y)$ and $(\tilde{x}, y, \tilde{x} \otimes y)$ are elements of $M_{m, d}^{\beta}(0, T)$. Let $f: \mathbb{R}^{m} \rightarrow \mathbb{R}^{d}$ be a twice continuously differentiable function such that the second derivative $f^{\prime \prime}$ is $\lambda$-Hölder continuous and $f^{\prime}$ and $f^{\prime \prime}$ are bounded, where $\lambda>\frac{1}{\beta}-2$. Then

$$
\begin{aligned}
& \left\|\int f\left(x_{r}\right) d y_{r}-\int f\left(\widetilde{x}_{r}\right) d y_{r}\right\|_{a, b, \beta} \\
\leq \quad & k H_{a, b}(y)\|x-\widetilde{x}\|_{a, b, \infty}+k H_{a, b}^{2}(y)\|x-\widetilde{x}\|_{a, b, \beta} \\
& +k H_{a, b}^{3}\|(x-\widetilde{x}) \otimes y\|_{a, b, 2 \beta},
\end{aligned}
$$

where

$$
\begin{aligned}
H_{a, b}^{1}(y)= & \|y\|_{a, b, \beta}\left\|f^{\prime}\right\|_{\infty}(b-a)^{\beta} \\
& +\left(\left\|f^{\prime \prime}\right\|_{\infty}+\left\|f^{\prime \prime}\right\|_{\lambda}\left(\|x\|_{a, b, \beta}^{\lambda}+\|\widetilde{x}\|_{a, b, \beta}^{\lambda}\right)(b-a)^{\beta \lambda}\right) \\
& \times\left(\|x \otimes y\|_{a, b, 2 \beta}+\left(\|x\|_{a, b, \beta}+\|\widetilde{x}\|_{a, b, \beta}\right)\|y\|_{a, b, \beta}\right)(b-a)^{\beta}, \\
(6.5)= & \left\|f^{\prime \prime}\right\|_{\infty}\left(\|x \otimes y\|_{a, b, 2 \beta}+\left(\|\widetilde{x}\|_{a, b, \beta}+\|x\|_{a, b, \beta}\right)\|y\|_{a, b, \beta}\right)(b-a)^{2 \beta} \\
& +\left\|f^{\prime}\right\|_{\infty}\|y\|_{a, b, \beta}(b-a)^{\beta}, \\
H_{a, b}^{2}(y)= & \left(\left\|f^{\prime}\right\|_{\infty}+\left\|f^{\prime \prime}\right\|_{\infty}\|\widetilde{x}\|_{a, b, \beta}(b-a)^{\beta}\right)(b-a)^{\beta} .
\end{aligned}
$$

Proof. Note that for any $a \leq \theta \leq r \leq b$ we can write

$$
\begin{aligned}
f\left(x_{r}\right)-f\left(x_{\theta}\right)-f^{\prime}\left(x_{\theta}\right)\left(x_{r}-x_{\theta}\right)-\left[f\left(\widetilde{x}_{r}\right)-f\left(\widetilde{x}_{\theta}\right)-f^{\prime}\left(\widetilde{x}_{\theta}\right)\left(\widetilde{x}_{r}-\widetilde{x}_{\theta}\right)\right] \\
=\int_{0}^{1}\left[f^{\prime}\left(x_{\theta}+z\left(x_{r}-x_{\theta}\right)\right)-f^{\prime}\left(x_{\theta}\right)\right]\left(x_{r}-x_{\theta}-\widetilde{x}_{r}+\widetilde{x}_{\theta}\right) d z \\
\quad+\int_{0}^{1}\left[f^{\prime}\left(x_{\theta}+z\left(x_{r}-x_{\theta}\right)\right)-f^{\prime}\left(\widetilde{x}_{\theta}+z\left(\widetilde{x}_{r}-\widetilde{x}_{\theta}\right)\right)-f^{\prime}\left(x_{\theta}\right)+f^{\prime}\left(\widetilde{x}_{\theta}\right)\right]\left(\widetilde{x}_{r}-\widetilde{x}_{\theta}\right) d z \\
=a_{1}-a_{2} .
\end{aligned}
$$

We have

$$
\left|a_{1}\right| \leq\left\|f^{\prime \prime}\right\|_{\infty}\|x\|_{a, b, \beta}\|x-\widetilde{x}\|_{a, b, \beta}(r-\theta)^{2 \beta} .
$$


For the term $a_{2}$ we make the decomposition

$$
\begin{aligned}
a_{2}=\left(\widetilde{x}_{r}-\right. & \left.\widetilde{x}_{\theta}\right) \int_{0}^{1} \int_{0}^{1}\left[\left(\widetilde{x}_{\theta}-x_{\theta}\right)+z\left(\widetilde{x}_{r}-\widetilde{x}_{\theta}-x_{r}+x_{\theta}\right)\right] \\
& \times f^{\prime \prime}\left(x_{\theta}+z\left(x_{r}-x_{\theta}\right)+t\left(\widetilde{x}_{\theta}-x_{\theta}\right)+t z\left(\widetilde{x}_{r}-\widetilde{x}_{\theta}-x_{r}+x_{\theta}\right)\right) d t d z \\
& \quad-\left(\widetilde{x}_{r}-\widetilde{x}_{\theta}\right)\left(\widetilde{x}_{\theta}-x_{\theta}\right) \int_{0}^{1} f^{\prime \prime}\left(x_{\theta}+t\left(\widetilde{x}_{\theta}-x_{\theta}\right)\right) d t \\
=\left(\widetilde{x}_{r}-\widetilde{x}_{\theta}-x_{r}+x_{\theta}\right)\left(\widetilde{x}_{r}-\widetilde{x}_{\theta}\right) & \\
\times & \int_{0}^{1} \int_{0}^{1} z f^{\prime \prime}\left(x_{\theta}+z\left(x_{r}-x_{\theta}\right)+t\left(\widetilde{x}_{\theta}-x_{\theta}\right)+t z\left(\widetilde{x}_{r}-\widetilde{x}_{\theta}-x_{r}+x_{\theta}\right)\right) d t d z \\
& -\left(\widetilde{x}_{r}-\widetilde{x}_{\theta}\right)\left(\widetilde{x}_{\theta}-x_{\theta}\right) \int_{0}^{1} \int_{0}^{1}\left[f ^ { \prime \prime } \left(x_{\theta}+z\left(x_{r}-x_{\theta}\right)+t\left(\widetilde{x}_{\theta}-x_{\theta}\right)\right.\right. \\
& \left.\left.+t z\left(\widetilde{x}_{r}-\widetilde{x}_{\theta}-x_{r}+x_{\theta}\right)\right)-f^{\prime \prime}\left(x_{\theta}+t\left(\widetilde{x}_{\theta}-x_{\theta}\right)\right)\right] d t d z .
\end{aligned}
$$

Thus,

$$
\begin{aligned}
\left|a_{2}\right| \leq & \left\|f^{\prime \prime}\right\|_{\infty}\|\widetilde{x}\|_{a, b, \beta}\|x-\widetilde{x}\|_{a, b, \beta}(r-\theta)^{2 \beta} \\
& +\left\|f^{\prime \prime}\right\|_{\lambda}\|\widetilde{x}\|_{a, b, \beta}\|x-\widetilde{x}\|_{a, b, \infty}\left(\|x\|_{a, b, \beta}^{\lambda}+\|x-\widetilde{x}\|_{a, b, \beta}^{\lambda}\right)(r-\theta)^{\beta(1+\lambda)} .
\end{aligned}
$$

As a consequence,

$$
\begin{aligned}
&\left|f\left(x_{r}\right)-f\left(x_{\theta}\right)-f^{\prime}\left(x_{\theta}\right)\left(x_{r}-x_{\theta}\right)-\left[f\left(\widetilde{x}_{r}\right)-f\left(\widetilde{x}_{\theta}\right)-f^{\prime}\left(\widetilde{x}_{\theta}\right)\left(\widetilde{x}_{r}-\widetilde{x}_{\theta}\right)\right]\right| \\
&(6.8) \leq \quad k I_{1}(r-\theta)^{\beta(1+\lambda)},
\end{aligned}
$$

where

$$
\begin{aligned}
I_{1}= & \left\|f^{\prime \prime}\right\|_{\infty}\left\{\|x\|_{a, b, \beta}+\|\widetilde{x}\|_{a, b, \beta}\right\}\|x-\widetilde{x}\|_{a, b, \beta} \\
& +\left\|f^{\prime \prime}\right\|_{\lambda}\|\widetilde{x}\|_{a, b, \beta}\left(\|x\|_{a, b, \beta}^{\lambda}+\|\widetilde{x}\|_{a, b, \beta}^{\lambda}\right)\|x-\widetilde{x}\|_{a, b, \infty} .
\end{aligned}
$$

On the other hand, we have

$$
\begin{aligned}
& D_{s+}^{2 \alpha-1} f^{\prime}(x)(r)-D_{s+}^{2 \alpha-1} f^{\prime}(\widetilde{x})(r) \\
= & \frac{1}{\Gamma(2-2 \alpha)}\left\{\frac{f^{\prime}\left(x_{r}\right)-f^{\prime}\left(\widetilde{x}_{r}\right)}{(r-s)^{2 \alpha-1}}\right. \\
& \left.+(2 \alpha-1) \int_{s}^{r} \frac{\left[f^{\prime}\left(x_{r}\right)-f^{\prime}\left(\widetilde{x}_{r}\right)-f^{\prime}\left(x_{\theta}\right)+f^{\prime}\left(\widetilde{x}_{\theta}\right)\right]}{(r-\theta)^{2 \alpha}} d \theta\right\} .
\end{aligned}
$$

Using the decomposition

$$
\begin{aligned}
& f^{\prime}\left(x_{r}\right)-f^{\prime}\left(\widetilde{x}_{r}\right)-f^{\prime}\left(x_{\theta}\right)+f^{\prime}\left(\widetilde{x}_{\theta}\right) \\
= & \int_{0}^{1} f^{\prime \prime}\left(x_{r}+t\left(\widetilde{x}_{r}-x_{r}\right)\right)\left(\widetilde{x}_{r}-x_{r}\right) d t-\int_{0}^{1} f^{\prime \prime}\left(x_{\theta}+t\left(\widetilde{x}_{\theta}-x_{\theta}\right)\right)\left(\widetilde{x}_{\theta}-x_{\theta}\right) d t,
\end{aligned}
$$

we obtain

$$
\begin{aligned}
& \left|D_{s+}^{2 \alpha-1} f^{\prime}(x)(r)-D_{s+}^{2 \alpha-1} f^{\prime}(\widetilde{x})(r)\right| \\
\leq & k(r-s)^{1-2 \alpha}\left\|f^{\prime \prime}\right\|_{\infty}\|x-\widetilde{x}\|_{s, r, \infty}+k(r-s)^{\beta-2 \alpha+1}\left\|f^{\prime \prime}\right\|_{\infty}\|x-\widetilde{x}\|_{s, r, \beta} \\
& +k(r-s)^{\beta \lambda-2 \alpha+1}\left\|f^{\prime \prime}\right\|_{\lambda}\left(\|x\|_{s, r, \beta}^{\lambda}+\|\widetilde{x}\|_{s, r, \beta}^{\lambda}\right)\|x-\widetilde{x}\|_{s, r, \infty} \\
(6.9)= & k I_{2}(r-s)^{1-2 \alpha}
\end{aligned}
$$


where

$$
\begin{aligned}
I_{2}= & \left(\left\|f^{\prime \prime}\right\|_{\infty}+\left\|f^{\prime \prime}\right\|_{\lambda}\left(\|x\|_{a, b, \beta}^{\lambda}+\|\widetilde{x}\|_{a, b, \beta}^{\lambda}\right)(b-a)^{\beta \lambda}\right)\|x-\widetilde{x}\|_{a, b, \infty} \\
& +\left\|f^{\prime \prime}\right\|_{\infty}\|x-\widetilde{x}\|_{a, b, \beta}(b-a)^{\beta} .
\end{aligned}
$$

Now using (3.18), (6.8), (6.9) we obtain

$$
\begin{aligned}
& \left|\int_{a}^{b}\left[f\left(x_{r}\right)-f\left(\widetilde{x}_{r}\right)\right] d y_{r}\right| \leq k\|y\|_{a, b, \beta} \\
& \quad \times\left(\int_{a}^{b}\left|f\left(x_{r}\right)-f\left(\widetilde{x}_{r}\right)\right|(r-a)^{-\alpha}(b-r)^{\alpha+\beta-1} d r\right. \\
& \left.\quad+I_{1} \int_{a}^{b}(r-a)^{\beta(1+\lambda)-\alpha}(b-r)^{\alpha+\beta-1} d r\right) \\
& \quad+k I_{2} \int_{a}^{b}(b-r)^{1-2 \alpha}\left|D_{b-}^{1-\alpha} \mathcal{D}_{b-}^{1-\alpha}(x \otimes y)(r)\right| d r \\
& \quad+\int_{a}^{b}\left|D_{a+}^{2 \alpha-1} f^{\prime}(\widetilde{x})(r)\right|\left|D_{b-}^{1-\alpha} \mathcal{D}_{b-}^{1-\alpha}((x-\widetilde{x}) \otimes y)(r)\right| d r .
\end{aligned}
$$

Finally, using (3.20) and (3.21), we get

$$
\begin{aligned}
& \left|\int_{a}^{b}\left[f\left(x_{r}\right)-f\left(\widetilde{x}_{r}\right)\right] d y_{r}\right| \leq k\|y\|_{a, b, \beta} \\
& \quad \times\left(\left\|f^{\prime}\right\|_{\infty}(b-a)^{2 \beta}\|x-\widetilde{x}\|_{a, b, \infty}+I_{1}(b-a)^{\beta(2+\lambda)}\right) \\
& \quad+k I_{2}\left(\|x \otimes y\|_{a, b, 2 \beta}+\|x\|_{a, b, \beta}\|y\|_{a, b, \beta}\right)(b-a)^{2 \beta} \\
& \quad+\left[\left\|f^{\prime}\right\|_{\infty}+\left\|f^{\prime \prime}\right\|_{\infty}\|\widetilde{x}\|_{a, b, \beta}(b-a)^{\beta}\right] \\
& \quad \times\left\{\|(x-\widetilde{x}) \otimes y\|_{a, b, 2 \beta}+\|x-\widetilde{x}\|_{a, b, \beta}\|y\|_{a, b, \beta}\right\}(b-a)^{2 \beta} .
\end{aligned}
$$

This implies (6.4).

The following corollary is a consequence of Propositions 3.4 and 3.5 ,

Corollary 6.5. Under the hypotheses of Proposition [3.5, we have

$$
\begin{array}{ll} 
& \left\|\int f\left(x_{r}\right) d y_{r}-\int \widetilde{f}\left(\widetilde{x}_{r}\right) d y_{r}\right\|_{a, b, \beta} \\
\leq & k H_{a, b}^{1, f}(y)\|x-\widetilde{x}\|_{a, b, \infty}+k H_{a, b}^{2, f}\|x-\widetilde{x}\|_{a, b, \beta}+k H_{a, b}^{3, f}\|(x-\widetilde{x}) \otimes y\|_{a, b, 2 \beta} \\
& +k\|f-\widetilde{f}\|_{\infty}\|y\|_{a, b, \beta}+k\left(\|x \otimes y\|_{a, b, 2 \beta}+\|x\|_{a, b, \beta}\|y\|_{a, b, \beta}\right) \\
\quad & \times\left(\left\|f^{\prime}-\widetilde{f}^{\prime}\right\|_{\infty}+\left\|f^{\prime}-\widetilde{f}^{\prime}\right\|_{\lambda}\|\widetilde{x}\|_{a, b, \beta}^{\lambda}(b-a)^{\lambda \beta}\right)(b-a)^{\beta} .
\end{array}
$$

\section{REFERENCES}

1. Coutin, L. and Lejay, A. Semi-martingales and rough paths theory. Electron. J. Probab. 10 (2005), 761-785. MR2164030 (2006i:60042)

2. Coutin, L. and Qian, Z. Stochastic analysis, rough path analysis and fractional Brownian motions. Probab. Theory Related Fields 122 (2002), 108-140. MR1883719 (2003c:60066) 
3. Friz, P. K. Continuity of the Itô-map for Hölder rough paths with applications to the support theorem in Hölder norm. In: Probability and partial differential equations in modern applied mathematics, 117-135, IMA Vol. Math. Appl., 140, Springer, New York, 2005. MR2202036 (2007f:60070)

4. Friz, P. and Victoir, N. Approximations of the Brownian rough path with applications to stochastic analysis. Ann. Inst. H. Poincaré 41 (2005), 703-724. MR 2144230(2007e:60018)

5. Gubinelli, M. Controlling rough paths. J. Funct. Anal. 216 (2004), 86-140. MR2091358 (2005k:60169)

6. Hu, Y. and Nualart, D. Differential equations driven by Hölder continuous functions of order greater than 1/2. Stochastic analysis and applications, 399-413, Abel symposium 2, Springer, 2007. MR2397797

7. Ledoux, M., Qian, Z., and Zhang, T. Large deviations and support theorem for diffusion processes via rough paths. Stochastic Process. Appl. 102 (2002), 265-283. MR 1935127 (2003m:60152)

8. Lejay, A. An introduction to rough paths. Lecture Notes in Math. 1832 (2003), 1-59. MR2053040 (2005e:60120)

9. Lyons, T. J. Differential equations driven by rough signals. I. An extension of an inequality of L. C. Young. Math. Res. Lett. 1 (1994), 451-464. MR1302388 (96b:60150)

10. Lyons, T. J. Differential equations driven by rough signals. Rev. Mat. Iberoamericana 14 (1998), 215-310. MR 1654527 (2000c:60089)

11. Lyons, T. J., Caruana, M., and Lévy, T. Differential equations driven by rough paths. Lecture Notes in Math. 1908. Springer-Verlag, 2007. MR.2314753

12. Lyons, T. J. and Qian, Z. Flow equations on spaces of rough paths. J. Funct. Anal. 149 (1997), 135-159. MR1471102 (99b:58241)

13. Lyons, T. and Qian, Z.M. System Control and Rough Paths. Clarendon Press, Oxford, 2002. MR2036784 (2005f:93001)

14. Millet, A. and Sanz-Solé, M. Large deviations for rough paths of the fractional Brownian motion. Ann. Inst. Henri Poincaré 42 (2006), 245-271. MR2199801(2007e:60027)

15. Nualart, D. and Răşcanu, A. Differential equations driven by fractional Brownian motion. Collect. Math. 53 (2002), 55-81. MR1893308(2003f:60105)

16. Samko S. G., Kilbas A. A. and Marichev O. I. Fractional Integrals and Derivatives. Theory and Applications. Gordon and Breach, 1993. MR1347689 (96d:26012)

17. Young, L. C. An inequality of the Hölder type connected with Stieltjes integration. Acta Math. 67 (1936) 251-282. MR 1555421

18. Zähle, M. Integration with respect to fractal functions and stochastic calculus. I. Prob. Theory Relat. Fields 111 (1998) 333-374. MR1640795 (99j:60073)

Department of Mathematics, University of Kansas, Lawrence, Kansas 66045-2142

E-mail address: hu@math.ku.edu

Department of Mathematics, University of Kansas, Lawrence, Kansas 66045-2142

E-mail address: nualart@math.ku.edu 